\title{
Scattering of gravity waves by potential vorticity in a shallow-water fluid
}

\author{
MARSHALL L. WARD ${ }^{1} \dagger$ AND WILLIAM K. DEWAR \\ ${ }^{1}$ Geophysical Fluid Dynamics Institute, Florida State University, Tallahassee, FL 32306, USA \\ ${ }^{2}$ Department of Oceanography, Florida State University, Tallahassee, FL 32306, USA
}

(Received 12 July 2009; revised 8 July 2010; accepted 10 July 2010;

first published online 8 October 2010)

The influence of a geostrophically balanced or potential vorticity (PV) containing background flow on the propagation of a coherent gravity wave is examined in a rotating shallow-water model. Over inertial time scales, we find that the gravity wave energy is scattered into other modes of similar wavelength, but with different directions of propagation. We attribute this response to nonlinear resonant interactions between the PV and gravity wave modes, despite the absence of any exchange of energy between the two, and show that the response is consistent with resonant triad theory. We first consider the scattering of a gravity wave mode due to a single PV mode, and compare the theoretical response to numerical solutions. This is followed by consideration of the propagation of a coherent gravity mode through a turbulent PV background. These results are expected to have relevance to the propagation of coherent internal tides in the open ocean.

Key words: ocean processes, quasi-geostrophic flows, wave-turbulence interactions

\section{Introduction}

Modelling efforts in geophysical fluid dynamics have contributed a vital dynamical context for the description of atmospheric and oceanic flows, and have provided accurate descriptions for the structure and evolution of major observed features such as large-scale geostrophically balanced currents, mesoscale eddies and inertiagravity waves. But the interaction of such structures across disparate length and time scales is still poorly understood and represents a limitation in our ability to simulate such phenomena. One of the strongest statements of scale separation is the assertion that geophysical flows have a slowly evolving balanced component that evolves independently of any high-frequency oscillations, at least in some approximate sense (Leith 1980; Lorenz 1980), and that the balanced flow can be described solely by the scalar potential vorticity (PV) field. Quasi-geostrophic theory (Charney 1948; Obukhov 1949; Pedlosky 1987) is an early example of a balanced model, and modern theories suggest that further refinement is possible (e.g. Warn et al. 1995; Ford, McIntyre \& Norton 2000; McIntyre \& Norton 2000; Reznik, Zeitlin \& Ben Jelloul 2001). Although these studies demonstrate that unbalanced gravity waves have a relatively weak impact on the balanced flow, most do not strictly imply that the gravity wave field evolves independently of the PV field. Rather, they leave open the

$\dagger$ Present address: Research School of Earth Sciences, Australian National University, Canberra, ACT 0200 Australia. Email address for correspondence: marshall.ward@anu.edu.au 
possibility that the PV field may act as a catalyst that can still alter the evolution of the gravity wave field. In this paper, we present evidence for a PV-induced scattering of coherent gravity waves in a shallow-water model.

The motivation of this study is primarily oceanographic. Geostrophically balanced ocean currents consist of steady jets spanning hundreds of kilometres and mesoscale eddies spanning tens of kilometres. Oceanic gravity waves, on the other hand, generally consist of high-frequency oscillations that contain a very sharp inertial peak, with wavelengths many times greater than the mesoscale eddy field, amid a complex spectrum whose wavelengths are least an order of magnitude smaller than the eddy field. Such strong separation in both length and time scales strengthen the assertion that there is little interaction between the balanced flow and the gravity wave field. But these observations describe the wind-forced gravity wave spectrum, and a major exception is the tidally forced spectrum. Since tidal forcing is dominated by just a few particular low frequencies, it is capable of producing a strongly coherent and monochromatic signal on length scales comparable to those of geostrophically balanced currents. Additionally, the barotropic tide has been identified as a significant energy source for deep ocean mixing, perhaps accounting for nearly half of the total abyssal power input, and may ultimately contribute to the maintenance of the meridional overturning circulation and ocean climate (Munk \& Wunsch 1998). There is a strong transfer of energy from the barotropic tide to the internal gravity wave field at major topographic sites (St. Laurent \& Garrett 2002), some of which dissipates locally and may contribute to hypothesized 'mixing hotspots' (Polzin et al. 1997). But a majority of the energy, perhaps as much as $95 \%$ in some regions, is converted to more coherent internal tides of low vertical mode that radiate into the open ocean (St. Laurent \& Nash 2004), with minimal energy lost to smaller-scale vertical modes (Olbers \& Pomphrey 1981). Numerical models suggest that the ocean can become filled with beams of internal waves that retain their coherence for long times (Simmons, Hallberg \& Arbic 2004) and may become unstable at latitudes near $29^{\circ}$, leading to enhanced open-ocean mixing (MacKinnon \& Winters 2005). While the ultimate fate of the tidally forced energy is currently uncertain, all of these results highlight the importance of a coherent low-mode internal wave field. This wave field would not only have length scales comparable to geostrophic currents, but would also be forced to propagate through such currents over substantial time intervals. The impact of these geostrophic currents on a tidally produced wave field is the primary motivation of this study.

The catalytic role of PV on gravity wave propagation has often been proposed in many studies (Duffy 1974; Warn 1986; Lelong \& Riley 1991; Bartello 1995; Dewar \& Killworth 1995), but more often in the context of its broader impact on the balanced flow. Most such assertions were based on resonant triad theory (Phillips 1960; Benney \& Saffman 1966), where pairs of waves can resonantly force a third wave through the nonlinear advection terms. Warn (1986) considered such resonances in the shallow-water model and concluded that PV modes can only be resonantly forced by other PV modes, while the resonant forcing of gravity waves requires the participation of another gravity wave and a single PV mode. In a similar nonrotating case, Lelong \& Riley (1991) identified the catalytic role of the PV mode in such interactions and proposed that they pushed the gravity wave spectrum towards a more isotropic state. The catalytic effect of the PV modes was observed in the numerical simulations of Waite \& Bartello (2006), where the introduction of PV forcing induced a transfer of energy to smaller vertical scales in their equilibrium gravity wave energy spectrum. Wave-PV interactions have also been explored in the propagation of 
near-inertial gravity waves through a PV background. Kunze (1985) applied raytracing theory to such propagation, treating the PV field as a passive medium, and identified a PV-induced trapping of such waves in regions of negative vorticity. These results were generalized by Young \& Ben Jelloul (1997), and were tested numerically in a turbulent PV field by Klein \& Llewellyn Smith (2001) and Klein, Llewellyn Smith \& Lapeyre (2004). Notably, Reznik et al. (2001) have argued against the existence of any PV-induced catalysis between smooth, localized distributions of PV and gravity wave fields in the asymptotic time limit $t \rightarrow \infty$. Also resonant interactions between gravity waves have been calculated for both deterministic and statistically equilibriated spectra (McComas \& Bretherton 1977; McComas \& Müller 1981; Müller et al. 1986; Caillol \& Zeitlin 2000; Lvov, Polzin \& Tabak 2004), but with only a limited focus on the role of PV in their evolution.

In this paper, we focus solely on the wave-PV interaction and demonstrate that PV can modify a coherent gravity wave field on inertial time scales. Although there is virtually no exchange of energy between the PV field and the gravity waves, the PV acts as a catalyst for the redistribution of energy within the gravity wave field. We also show that this interaction is consistent with resonant triad theory. We avoid a statistical approach and focus on the deterministic evolution of the wave field, which allows us to make more straightforward comparisons between theoretical predictions and numerical model simulations. In $\S 2$, the shallow-water equations are formulated in terms of the normal modes, paying particular attention to the representation of the nonlinear terms. Then we apply a multiple-scale analysis that isolates the resonant triad interactions from the nonlinear terms, providing the general framework for an evolution equation of the wave amplitudes on inertial time scales. Section 3 looks at the interactions between individual modes in more detail. We show that resonances within the gravity wave field can only exist if an appropriate PV field is also present. This permits us to construct a modulational equation for the gravity wave amplitudes as they propagate through an active PV field. We then provide numerical confirmation of resonant PV-gravity wave interaction in a shallow-water model, and compare the numerical solution to the theoretical predictions. In $\S 4$, we attempt to model more realistic oceanographic conditions by placing a singleharmonic gravity wave in an active geostrophic turbulence field. We show that energy is only transferred to other resonant modes, and that the rate of transfer depends on the wavenumber and background PV intensity. We conclude in $\S 5$ by scaling our results to reasonable oceanographic values and assessing the relevance of PV-induced scattering on propagation of the internal tide.

\section{Methods}

Our analysis of PV-induced scattering of gravity waves is based on solutions from resonant triad theory and on numerical solutions of the shallow-water equations. In $\S 2$, we first construct our equations of motion in terms of resonantly coupled modes. This is followed by a specification of the model and numerical methods used in this paper.

\subsection{Modal representation of the shallow-water equations}

The resonant interactions between gravity waves and PV flows are examined using the eigenmode analysis of Warn (1986), which reconstructs the equations of motion in terms of the shallow-water eigenmodes. A similar method has been recently used 
by Remmel \& Smith (2009), although resonant triad interactions were not considered. Details can be found in Appendix A, and we highlight the key results in this section. In their simplest form, the equations for a rotating $f$-plane shallow-water model are

$$
\begin{aligned}
& \frac{\mathrm{D} \boldsymbol{u}}{\mathrm{D} t}+f_{0} \hat{z} \times \boldsymbol{u}=-g \nabla h, \\
& \frac{\mathrm{D} h}{\mathrm{D} t}+\left(H_{0}+h\right) \nabla \cdot \boldsymbol{u}=0,
\end{aligned}
$$

where $\boldsymbol{u}=(u, v)$ is the horizontal velocity, $H_{0}$ is the equilibrium fluid depth, $h$ is the perturbation fluid height, $f_{0}$ is the Coriolis parameter, $g$ is the (reduced) gravitational acceleration and $\mathrm{D} / \mathrm{D} t \equiv \partial_{t}+\boldsymbol{u} \cdot \nabla$ is the advective derivative. We first apply the following re-scalings:

$$
t \rightarrow T t, \quad \boldsymbol{x} \rightarrow L \boldsymbol{x}, \quad \boldsymbol{u} \rightarrow \epsilon c_{0} \boldsymbol{u}, \quad h \rightarrow \epsilon H_{0} h
$$

where $c_{0}=\sqrt{g H_{0}}$ is the non-rotational gravity wave speed and $\epsilon$ represents a rescaling of $\boldsymbol{u}$ and $h$ to $O(1)$ quantities relative to $c_{0}$ and $H_{0}$, respectively, while $L$ and $T$ represent length and time scales of observational interest, respectively. The re-scaled equations of motion are

$$
\begin{aligned}
u_{t}-f v+c h_{x} & =-\epsilon \boldsymbol{u} \cdot \nabla u, \\
v_{t}+f u+c h_{y} & =-\epsilon \boldsymbol{u} \cdot \nabla v, \\
h_{t}+c\left(u_{x}+v_{y}\right) & =-\epsilon \nabla \cdot(h \boldsymbol{u}),
\end{aligned}
$$

where $f=f_{0} T$ and $c=c_{0} T / L$. When viewed as a geophysical problem, the Rossby number is $R o=\epsilon / f$ and the Froude number is $F r=\epsilon / c$, while $\lambda=c / f$ is the deformation radius relative to $L$. We have also adopted the traditional assumption that $|h| / H_{0}$ and $|\boldsymbol{u}| / c_{0}$ are similar in magnitude. Although no fixed length and time scales are assumed, we generally do not deviate far from the deformation radius and can usually assume that $\lambda \approx 1$. As in most geophysical applications, we typically assume that $F r, R o \ll 1$, which corresponds to $\epsilon \ll 1$ in most cases, but we also presume that $\epsilon$ is not negligible and that nonlinear effects are relevant to the long-term evolution of the system.

The shallow-water equations (2.3) can be schematically represented as

$$
\frac{\partial \Phi}{\partial t}+\mathrm{i} H \Phi=\epsilon \mathscr{N}(\Phi)
$$

where $\Phi=(u, v, h)^{\mathrm{T}}$ is the solution in terms of physical variables and $H$ is a Hermitian linear operator acting on $\Phi$. The nonlinearity is represented by $\mathscr{N}$ and depends quadratically on the elements of $\Phi$. For the idealized case of $\epsilon=0$, the system is linear and is driven entirely by wave propagation of three unique classes of eigenmodes. Each mode is denoted by its wavenumber $\boldsymbol{k}$ and mode type $s \in\{0,+,-\}$ and has a corresponding eigenvalue $\omega_{k}^{s}$ and eigenmode $\Psi_{k}^{s}$. One mode has eigenvalue $\omega_{k}^{0}=0$ and corresponds to a static geostrophically balanced flow with an associated $\mathrm{PV}$ field, defined as

$$
Q=\nabla^{2} h-\lambda^{-2} h,
$$

where $Q$ is the linearized PV. We refer to these as PV modes. The other two eigenmodes are propagating gravity waves with eigenvalues $\omega_{k}^{ \pm}= \pm \sqrt{f^{2}+c^{2}|\boldsymbol{k}|^{2}}$, and contain no PV. 
Since the eigenmodes are orthogonal, the full nonlinear solution $\Phi$ can be stated in terms of the mode amplitudes. That is, $\Phi$ can be represented as

$$
\Phi_{k}(t)=\sum_{s} A_{k}^{s}(t) \Psi_{k}^{s}
$$

where $A_{k}^{s}$ are the mode amplitudes. Furthermore, the equations of motion can be decomposed into a set of equations for each mode wavenumber $\boldsymbol{k}$ and mode type $s$, of the form

$$
\frac{\partial A_{k}^{s}}{\partial t}+\mathrm{i} \omega_{k}^{s} A_{k}^{s}=\epsilon N_{k}^{s}
$$

where $N_{k}^{s}$ is the projection of the nonlinear terms onto the eigenmode $\Psi_{k}^{s}$. The most challenging step is to represent $N_{k}^{s}$ in terms of the modal amplitudes $A_{k}^{s}$. The procedure is algebraically complex, but the general functional form is a quadratic dependence on the mode amplitudes, which is a direct reflection of the quadratic dependence on the physical variables. The modal projection of the nonlinearity is

$$
N_{1}^{i}=\sum_{j k} \int_{23} \Gamma_{123}^{i j k} A_{2}^{j} A_{3}^{k} \mathrm{~d} \boldsymbol{k}_{23},
$$

where numerical subscripts $1,2,3$ refer to modal variables $\boldsymbol{k}_{1}, \boldsymbol{k}_{2}, \boldsymbol{k}_{3}$. In this form, the nonlinearity is interpreted as a series of wave triad interactions. Specifically, each mode $\boldsymbol{k}_{1}$ is forced by every possible combination of mode pairs $\boldsymbol{k}_{2}$ and $\boldsymbol{k}_{3}$ that satisfy the constraint $\boldsymbol{k}_{1}=\boldsymbol{k}_{2}+\boldsymbol{k}_{3}$. The intensity of each triad is weighted by the interaction coefficients $\Gamma_{123}^{i j k}$, which also contains the triad constraint. Integration is over all possible triads, and further summed over all possible wave types.

The modal representation of the equations of motion is

$$
\frac{\partial A_{1}^{i}}{\partial t}+\mathrm{i} \omega_{1}^{i} A_{1}^{i}=\epsilon \sum_{j k} \int_{23} \Gamma_{123}^{i j k} A_{2}^{j} A_{3}^{k} \mathrm{~d} \boldsymbol{k}_{23} .
$$

This equation is equivalent to the original representation of the equations of motion (2.3). Even though the modal representation can be more difficult to interpret physically, the main advantage of this form is that everything is expressed in terms of interactions between normal modes. This allows us to identify and retain the triads that have a persistent and dominant impact on wave propagation.

\subsection{Perturbative solutions to the nonlinear equations}

Using the reformulated equations of motion (2.9), we now seek a perturbative solution that is dominated by wave propagation but preserves the nonlinearity as a weak wave-wave interaction. A regular perturbative solution of the form

$$
A_{1}^{i}=A_{1}^{i(0)}+\epsilon A_{1}^{i(1)}+\epsilon^{2} A_{1}^{i(2)}+\cdots,
$$

will ultimately fail to be of use over long times, due to resonant forcing at higher orders by lower-order terms. For example, the $O(1)$ equation is equivalent to the linear problem and the solution is $A_{1}^{i(0)}=a_{1}^{i} \mathrm{e}^{-\mathrm{i} \omega_{1}^{i} t}$ for initial condition $a_{1}^{i}$. For each successive order, the linear terms are essentially unchanged and the nonlinear terms act as an external forcing induced by the lower-order solutions. At $O(\epsilon)$, the equation for $A_{1}^{i(1)}$ is

$$
\frac{\partial}{\partial t} A_{1}^{i(1)}+\mathrm{i} \omega_{1}^{i} A_{1}^{i(1)}=\sum_{j k} \int_{23} \Gamma_{123}^{i j k} a_{2}^{j} a_{3}^{k} \mathrm{e}^{-\mathrm{i}\left(\omega_{2}^{j}+\omega_{3}^{k}\right) t} \mathrm{~d} \boldsymbol{k}_{23},
$$


where the integration is over all wave triads $\left(\boldsymbol{k}_{1}, \boldsymbol{k}_{2}, \boldsymbol{k}_{3}\right)$ that satisfy $\boldsymbol{k}_{1}=\boldsymbol{k}_{2}+\boldsymbol{k}_{3}$. Each mode $\boldsymbol{k}_{1}$ oscillates at its natural frequency $\omega_{1}$, while each triad produces a forcing frequency of $\omega_{2}+\omega_{3}$. If any of these triads also satisfy $\omega_{1}=\omega_{2}+\omega_{3}$, so that the forcing frequency matches the natural frequency, then a resonance emerges and $A_{1}^{i(1)}$ will grow linearly with time. Similar resonances at higher orders lead to amplitudes of almost arbitrary magnitude at each order of $\epsilon$, leading to a perturbative structure that is no longer well-ordered for times $t \gg 1$.

A multiple time scale analysis can be used to mitigate this secular growth. If we introduce a slow time scale $\tau \equiv \epsilon t$ and interpret the time dependence of the amplitudes as $A_{1}^{i}(t, \tau)$, then the original equation of motion (2.9) becomes

$$
\frac{\partial A_{1}^{i}}{\partial t}+\epsilon \frac{\partial A_{1}^{i}}{\partial \tau}+\mathrm{i} \omega_{1}^{i} A_{1}^{i}=\epsilon \sum_{j k} \int_{23} \Gamma_{123}^{i j k} A_{2}^{j} A_{3}^{k} \mathrm{~d} \boldsymbol{k}_{23} .
$$

The $O(1)$ solution for each mode is $A_{1}^{i(0)}=a_{1}^{i}(\tau) \mathrm{e}^{-\mathrm{i} \omega_{1}^{i} t}$ where our integration constants $a_{1}^{i}(\tau)$ are now unknown functions with the initial conditions $a_{1}^{i}$. The slow modulational evolution of $a_{1}^{i}$ is determined by the $O(\epsilon)$ dynamics, whose new equation is the same as (2.11) except for the additional modulational term, $\partial a_{1}^{i} / \partial \tau$. If we decompose the integral into its resonant and non-resonant parts, then the solution is

$$
\begin{aligned}
A_{1}^{i(1)}+t \frac{\partial a_{1}^{i}}{\partial \tau} \mathrm{e}^{-\mathrm{i} \omega_{1}^{i} t}=t \mathrm{e}^{-\mathrm{i} \omega_{1}^{i} t} & \sum_{\substack{j k \\
\omega_{123}^{i j k}=0}} \int_{23} \Gamma_{123}^{i j k} a_{2}^{j} a_{3}^{k} \mathrm{~d} \boldsymbol{k}_{23} \\
& +\sum_{\substack{j k \\
\omega_{123}^{j j k} \neq 0}} \int_{23} \frac{\Gamma_{123}^{i j k}}{\mathrm{i} \omega_{123}^{i j k}} a_{2}^{j} a_{3}^{k} \mathrm{e}^{-\mathrm{i}\left(\omega_{2}^{i}+\omega_{3}^{k}\right) t} \mathrm{~d} \boldsymbol{k}_{23}+C_{1}^{i(1)} \mathrm{e}^{-\mathrm{i} \omega_{1}^{i} t},
\end{aligned}
$$

where $\omega_{123}^{i j k}=\omega_{1}^{i}-\omega_{2}^{j}-\omega_{3}^{k}$ and $C_{1}^{i(1)}$ is a new integration constant. If there are any triads which satisfy the resonance condition $\omega_{123}^{i j k}=0$, then the emerging resonances at $t \rightarrow \infty$ are eliminated by the constraint

$$
\frac{\partial a_{1}^{i}}{\partial \tau}=\sum_{\substack{j k \\ \text { ijk } \\ \omega_{123}=0}} \int_{23} \Gamma_{123}^{i j k} a_{2}^{j} a_{3}^{k} \mathrm{~d} \boldsymbol{k}_{23}
$$

This equation describes the persistent dynamics of the $O(1)$ wave amplitudes. When the constraint is applied, the perturbation amplitude remains bounded and is driven by the non-resonant triads, given by

$$
A_{1}^{i(1)}(t, \tau)=C_{1}^{i(1)} \mathrm{e}^{-\mathrm{i} \omega_{1}^{i} t}+\sum_{\substack{j k \\ \omega_{123}^{j j k} \neq 0}} \int_{23} \frac{\Gamma_{123}^{i j k}}{\mathrm{i} \omega_{123}^{i j k}} a_{2}^{j}(\tau) a_{3}^{k}(\tau) \mathrm{e}^{-\mathrm{i}\left(\omega_{2}^{j}+\omega_{3}^{k}\right) t} \mathrm{~d} \boldsymbol{k}_{23} .
$$

Although $O\left(\epsilon^{2}\right)$ resonances may introduce a time dependence to $C_{1}^{(1)}$ just as $O(\epsilon)$ resonances produce a time dependence in $a_{1}^{i}$, it is sufficient for our purposes to assume that $C_{1}^{(1)}$ is constant to $O(\epsilon)$ and has the value

$$
C_{1}^{i(1)}=-\sum_{\substack{j k \\ \omega_{123}^{i k} \neq 0}} \int_{23} \frac{\Gamma_{123}^{i j k}}{\mathrm{i} \omega_{123}^{i j k}} a_{2}^{j}(0) a_{3}^{k}(0) \mathrm{d} \boldsymbol{k}_{23}
$$


One of the strongest contributions of resonant triad theory is its ability to highlight the limitations of linear wave dynamics. The emergent resonances are more often an indication of processes that cannot be accurately described by the wave field, and of a transition to a more inertially driven flow. The isolation of these triads and the use of the modulational equation (2.14) provides us with a starting point in understanding the slower persistent dynamics caused by nonlinear wave interactions.

Before we consider the resonant triad analysis of the shallow-water model, we briefly comment on near-resonant interactions. Although resonant triad theory is a powerful tool in the analysis of systems with multiple time scales, it is based on the idea that only resonant interactions, which diverge as $t \rightarrow \infty$, can have a persistent effect on a flow. In practice, this constraint is too strong and is not consistent with the assumed perturbative structure (2.10). In fact, any process that causes the amplitude to exceed $O\left(\epsilon^{-1}\right)$ values will invalidate our perturbative expansion. For example, if the triad forcing has a frequency $\omega_{2}+\omega_{3}$ and is applied to a mode with a natural frequency $\omega_{1}$, then the $O(\epsilon)$ response to this forcing will be proportional to $\left(\omega_{123}\right)^{-1}$, as seen in our solution $(2.15)$ to $A_{1}^{i(1)}(t, \tau)$. So any nearly resonant triad with a frequency difference $\omega_{123}$ that is $O(\epsilon)$ can, in principle, make a persistent contribution to the flow and should properly be interpreted as a contribution to the system on inertial time scales. Although we do not attempt to assess the impact of near-resonance in this study, we note that it can have a strong influence on the flow, evidence of which can be seen in $\S 4$.

\subsection{Numerical analysis}

The dynamical ideas and theoretical results in this paper are supported by numerical solutions of the shallow-water model. We solve the shallow-water equations as stated in (2.3) with parameters $c=f=1$ and $\epsilon=0.1$, so that times are reported relative to $f^{-1}$ and lengths are relative to the deformation radius, $c / f$. The domain is square periodic with a side length of $L=20 \pi$ on a $256 \times 256$ resolution grid, yielding a spatial resolution of $\Delta x \approx 0.25$ and a corresponding modal resolution of $\Delta k=0.1$. The equations are integrated numerically using a leapfrog trapezoidal time step (initialized with a second-order Runge-Kutta time step) of size $\Delta t=10^{-2}$. Derivatives are calculated pseudospectrally using a 2/3-dealiasing filter (Orszag 1971), which removes energy from modes $\boldsymbol{k}=(k, l)$ with wavenumber components $k, l>8.5$ after each time step. Model initialization is specified in terms of PV and gravity wave mode amplitudes $\left\{a_{k}^{0}, a_{k}^{ \pm}\right\}$, which are projected onto the physical variables $\{u, v, h\}$ using the eigenvectors (A 10).

\section{Single-triad interactions}

Using the framework established in $\$ 2$, we now consider PV-gravity wave interaction in terms of resonant triads. We begin by looking at the resonant condition $\omega_{1}^{i}=\omega_{2}^{j}+\omega_{3}^{k}$ for each triad type $(i, j, k)$. While most of these conclusions have been previously reported for the shallow-water model (Duffy 1974; Warn 1986) and similar geophysical systems (Lelong \& Riley 1991; Bartello 1995), the emphasis has traditionally been on the response of the PV field. Since our focus is on the gravity wave field, we perform an analysis similar to these previous studies and consider the contribution of each class of triad interactions to the gravity wave field. As in Bartello (1995), we also discuss the role of rotation and the possibility of near-resonance for each triad type. This is followed by an explicit solution of the modulational equation for a single-triad interaction, with a comparison to primitive equation calculations. 


\subsection{Resonant forcing of potential-vorticity modes}

We first consider the forcing of PV modes. The interaction of three PV modes, denoted by $(0,0,0)$, is trivially resonant since $\omega_{k}^{0}=0$ for all $\boldsymbol{k}$. None of the PV interactions can be dismissed as non-resonant and, all other things being equal, every triad is important. Only the details of the flow can dictate whether particular length scales or classes of triads are dominant.

A single PV and gravity mode acting on a PV mode in a $(0,0, \pm)$ triad is neither resonant nor near-resonant, since

$$
\left|\omega_{123}^{00 \pm}\right|=\sqrt{f^{2}+c^{2} K^{2}} \geqslant|f| \gg \epsilon .
$$

The absence of near-resonant $(0,0, \pm)$ interactions is a consequence of rapid rotation $(f \geqslant 1)$. If the system were not rotating, then the resonance condition would reduce to $\omega_{123}^{00 \pm}= \pm c K$, and near-resonance would be a concern for large-scale gravity waves where the wavenumbers $K$ are $O(\epsilon)$. When the system is rapidly rotating, it introduces an $O(1)$ spectral gap between PV and gravity modes that suppresses these interactions.

The final possibility is of two gravity modes acting on a PV mode in a $(0, \pm, \pm)$ interaction. This can only form a resonant triad if $\omega_{2}^{j}+\omega_{3}^{k}=0$, such as a gravity mode interacting with its conjugate in a real-valued signal. However, it is shown in Appendix A that $\Gamma_{123}^{0 \pm \pm}=0$ for all triads of this type, resonant or otherwise (Warn 1986; Lelong \& Riley 1991), and so they can never contribute to PV evolution.

From these results, we conclude that PV modes only interact with other PV modes on the modulational time scale $\tau$ and that the amplitudes evolve according to

$$
\frac{\partial a_{1}^{0}}{\partial \tau}=\int_{23} \Gamma_{123}^{000} a_{2}^{0} a_{3}^{0} \mathrm{~d} \boldsymbol{k}_{23} .
$$

This is the spectral representation of the quasi-geostrophic equation

$$
\frac{\partial}{\partial \tau}\left(\nabla^{2} \bar{h}-\lambda^{-2} \bar{h}\right)+\lambda J\left(\bar{h}, \nabla^{2} \bar{h}-\lambda^{-2} \bar{h}\right)=0
$$

where $\bar{h}$ is the geostrophically balanced part of $h$ (see Appendix B). In other words, resonant triad theory can be used to justify the removal of gravity waves and to establish quasi-geostrophic theory. This conclusion has been previously reported by Warn (1986) and was reproduced by Dewar \& Killworth (1995) in spatial coordinates. For our purposes, it means that the background PV field evolves independently of the gravity wave field over inertial time intervals of size $\Delta t=O\left(\epsilon^{-1}\right)$ and can be interpreted as an external forcing.

\subsection{Resonant forcing of gravity waves}

We now follow the same argument for the resonant forcing of gravity waves. Triads of type $( \pm, 0,0)$, or two PV modes acting on a gravity wave, are neither resonant nor nearly resonant for the same reasons as the $(0,0, \pm)$ triads. The absence of near-resonance is again a consequence of background rotation.

The most crucial interactions for our purposes are of type $( \pm, 0, \pm)$, where a PV mode and a gravity wave combine to force a second gravity wave. These triads become resonant when

$$
\omega_{123}^{ \pm 0 \pm}=\sqrt{f^{2}+c^{2} K_{1}^{2}}-\sqrt{f^{2}+c^{2} K_{3}^{2}}=0,
$$

which reduces to $K_{1}=K_{3}$. In other words, gravity waves of equal wavelength but different direction can resonantly interact, as long as there is a corresponding PV 
mode to bridge the waves and form a triad. These triads represent a special case of the resonant cone surface depicted in Lelong \& Riley (1991) along a fixed vertical mode. This result holds for both rotating and non-rotating flows, and these are the only triads capable of resonantly forcing gravity waves.

Triads of type $( \pm, \pm, \pm)$ consisting of three gravity waves are always non-resonant. This can be shown by substituting the resonant constraint $\omega_{1}^{ \pm}=\omega_{2}^{ \pm}+\omega_{3}^{ \pm}$into the triad constraint $\boldsymbol{k}_{1}=\boldsymbol{k}_{2}+\boldsymbol{k}_{3}$. If $\phi$ is the angle between $\boldsymbol{k}_{2}$ and $\boldsymbol{k}_{3}$, then the wavenumber $K_{3}$ is related to $K_{2}$ and $\phi$ by the quadratic equation

$$
\left(1+\tilde{K}_{2}^{2} \sin ^{2} \phi\right) \tilde{K}_{3}^{2}+\left(\tilde{K}_{2} \cos \phi\right) \tilde{K}_{3}+\left(\frac{3}{4}+\tilde{K}_{2}^{2}\right)=0
$$

where $\tilde{K}_{j}=(c / f) K_{j}$. However, there are no real-valued solutions to $K_{3}$, since the discriminant is always negative. Since $K_{3}$ must be real, we conclude that there are no resonant triads of type $( \pm, \pm, \pm)$ in the shallow-water model. This does not preclude interactions between different vertical modes in a stratified flow, which play a significant role in small-scale gravity wave interactions, but this subject is outside the context of this paper.

Despite the absence of resonance in $( \pm, \pm, \pm)$ triads, a much more realistic concern is near-resonant interactions, particularly the self-interaction and frontal steepening of small-scale gravity waves. For the self-interaction of mode $\boldsymbol{k}$ onto $2 \boldsymbol{k}$, the triad frequency difference is $\omega_{123}^{ \pm \pm \pm}=(3 / 4)\left(f^{2} / c\right) K^{-1}+O\left(K^{-3}\right)$ for $K \gg 1$, and selfinteraction becomes near-resonant for small-scale waves satisfying $K \approx \epsilon^{-1}$. As with other wave-PV triads, rotation tends to suppress such resonances. For a non-rotating flow, all gravity wave self-interaction is exactly resonant, regardless of length scale. Rotation changes this to a conditional near-resonant process that allows large-scale gravity waves to remain approximately coherent. Despite this suppression, however, most of the deviations from resonant triad theory in our numerical calculations are due to these self-interactions.

By restricting ourselves to the resonant triads of type $( \pm, 0, \pm)$ and neglecting near-resonant effects, the equation of motion for the gravity wave field reduces to

$$
\frac{\partial a_{1}^{+}}{\partial \tau}=\int_{K_{2}=K_{3}} \Gamma_{123}^{+0+} a_{2}^{0} a_{3}^{+} \mathrm{d} \boldsymbol{k}_{23} .
$$

A similar equation for $a_{k}^{-}$also exists, but these amplitudes can be inferred from the relation $a_{k}^{-}=\left(a_{-k}^{+}\right)^{*}$. We argue that this equation describes the primary evolution of a gravity wave field in a PV background. Near-resonant self-interaction of gravity waves can be safely dismissed if the PV amplitudes are significantly larger than the gravity wave amplitudes, or if the wavelengths are large compared to deformation radius. But over long time intervals, it may be necessary to incorporate these processes into the equation.

One notable consequence of resonant triad theory is that the PV amplitudes $a_{k}^{0}$ evolve independently of the gravity waves, reducing the modulational equation for the gravity wave amplitudes to a system of linear integral equations. Solutions can be constructed by first solving (3.2) for the PV modes, and then using these solutions to solve (3.6) for the gravity modes.

\subsection{Resonant interaction details}

Resonant triad theory predicts that gravity wave energy is preferentially scattered to other waves of equal wavelength, and that this scattering is catalyzed by PV modes. The process is driven by $( \pm, 0, \pm)$ triads, and the intensity of these interactions 
depends on the amplitudes of the modes and the interaction coefficients $\Gamma_{123}^{ \pm 0 \pm}$. The modal distribution is wholly dependent on the particular flow of interest, but the structure of $\Gamma_{123}^{ \pm 0 \pm}$ can also have a significant impact.

If we impose the resonant constraint of equal gravity wavelengths, so that $K_{1}=K_{3}$, then the $( \pm, 0, \pm)$ interaction coefficient can be specified by the incident gravity wavenumber magnitude $K$ and the relative angle of scattering $\theta$ between $\boldsymbol{k}_{1}$ and $\boldsymbol{k}_{3}$. The PV mode is inferred from the triad constraint $\boldsymbol{k}_{2}=\boldsymbol{k}_{1}-\boldsymbol{k}_{3}$. Using results in Appendix A, the resonant interaction coefficient is

$$
\Gamma_{R e s}^{ \pm 0 \pm}(K, \theta)=\frac{c K^{2}}{4 \Omega^{2}} \frac{\left(\left(f^{2}+\Omega^{2}\right) \sin 2 \theta-f^{2} \sin \theta\right) \pm \mathrm{i} f \Omega(2 \cos 2 \theta-\cos \theta)}{\sqrt{f^{2}+4 c^{2} K^{2} \sin ^{2}(\theta / 2)}} .
$$

where $\Omega=\sqrt{f^{2}+c^{2} K^{2}}$. The amplitude of $\Gamma_{R e s}^{+0+}$ for various regimes is shown in figure 1 .

For large-scale waves $(K \ll c / f)$, corresponding to the near-inertial spectrum, the interaction coefficient amplitude is

$$
\Gamma_{R e s}^{ \pm 0 \pm}(K, \theta) \sim \pm \mathrm{i} \frac{c}{4 f} K^{2}\left(2 \mathrm{e}^{\mp 2 \mathrm{i} \theta}-\mathrm{e}^{\mp \mathrm{i} \theta}\right),
$$

and is shown for $K=0.1 \mathrm{f} / \mathrm{c}$ in figure $1(a)$. The weakest response is in the direction of the wave, while the strongest response is in the opposite direction. The functional form is entirely due to rotational effects. However, we note that $\Gamma_{\text {Res }}$ is generally very weak in this regime, and the corresponding nonlinear interactions are expected to be negligible.

For small-scale waves $(K \gg c / f), \Gamma_{\text {Res }}$ is

$$
\Gamma_{\operatorname{Res}}^{ \pm 0 \pm}(K, \theta) \sim \frac{1}{8} K \frac{\sin 2 \theta}{|\sin (\theta / 2)|},
$$

except near integral angles $\theta_{n}=(\pi / 2) n$, where $\Gamma_{\text {Res }}$ behaves like

$$
\begin{aligned}
\Gamma_{R e s}^{+0+}(K, 0) & \sim \mathrm{i} \frac{K}{4}, \\
\Gamma_{R e s}^{+0+}\left(K, \pm \frac{\pi}{2}\right) & \sim \mp \mathrm{i} \frac{1}{2 \sqrt{2}} \frac{f}{c}, \\
\Gamma_{R e s}^{+0+}(K, \pi) & \sim \mathrm{i} \frac{3}{8} \frac{f}{c},
\end{aligned}
$$

and is shown for $K=10 \mathrm{f} / \mathrm{c}$ in figure $1(\mathrm{c})$. The plot suggests that the scattering of small-scale waves will tend to be in the same direction as the propagating wave, and is likely to spread to a number of modes along the same general direction. We also note that the expression (3.9) for $\Gamma$ in the small-scale limit is identical to the non-rotating resonant interaction coefficient, showing that the effects of rotation become negligible as $K$ becomes large $(K \gg c / f)$.

These results suggest that there may be noticeable inhomogeneities in the response of any purely harmonic gravity mode that enters a region of strong PV with a relatively uniform spectral distribution. The response of large-scale modes may tend to produce a backscatter in the opposite direction to propagation, while small-scale waves may tend to produce a stronger, if less coherent, response in the direction of propagation. Waves with $K \sim c / f$ should tend to produce a more homogeneous scattering response. However, we stress that the eventual modal response is dictated 

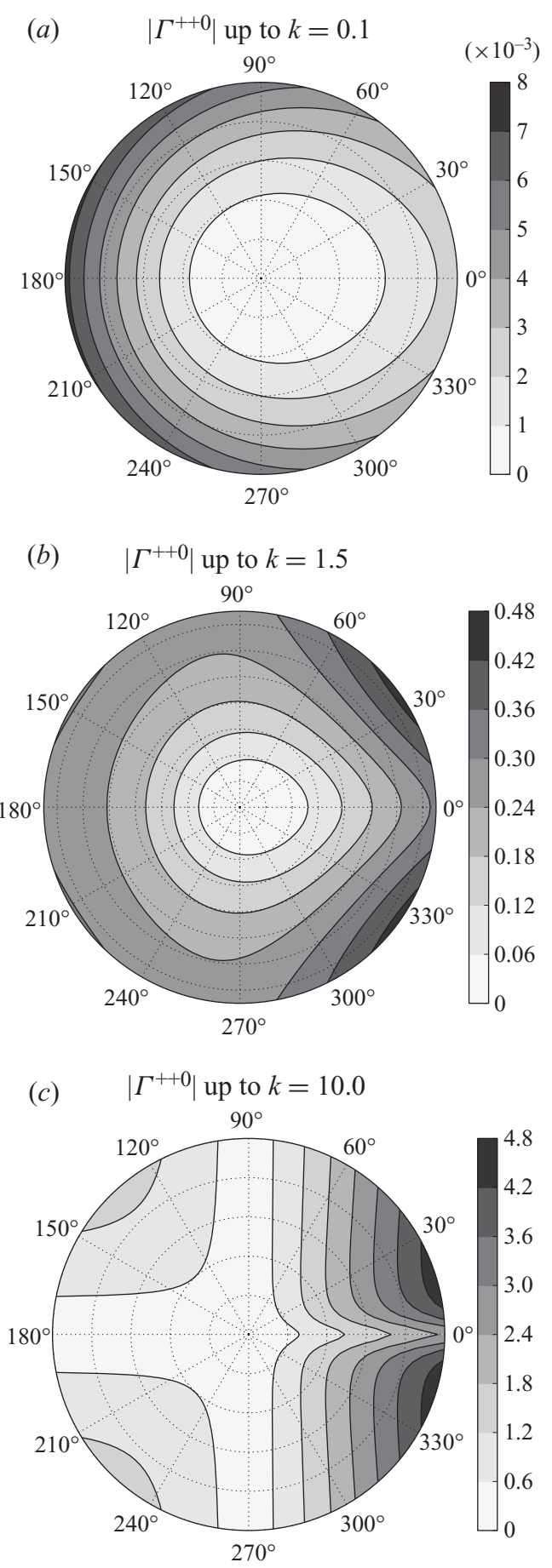

FIGURE 1. Structure of resonant interaction coefficients $\Gamma_{123}^{+0+}$ for $c=f=1$. The incident gravity mode $\boldsymbol{k}_{3}$ is assumed to point in the $0^{\circ}$ direction, so that the angle corresponds to the direction of the scattered mode $\boldsymbol{k}_{1}$. 


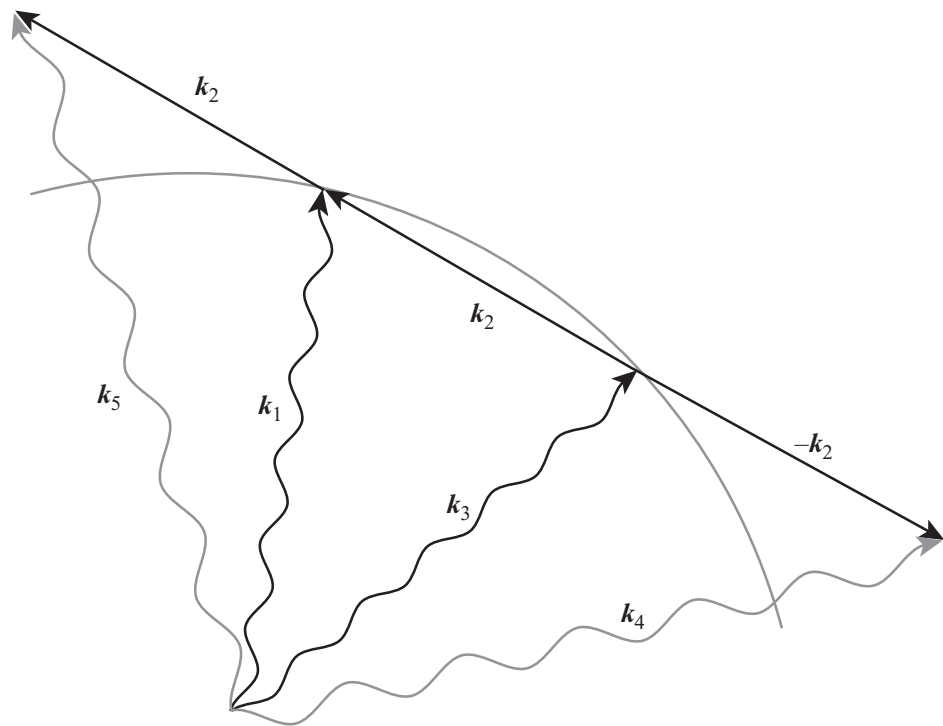

FIGURE 2. The single triad analysed in $\S 3.4$, along with the initial non-resonant responses, is depicted. Energy from gravity mode $\boldsymbol{k}_{3}$ can be scattered into either $\boldsymbol{k}_{1}$ or $\boldsymbol{k}_{4}$ through the PV modes $\pm \boldsymbol{k}_{2}$. Gravity mode $\boldsymbol{k}_{1}$ can similarly scatter its energy to either $\boldsymbol{k}_{3}$ or $\boldsymbol{k}_{5}$. The circular arc denotes the spectrum of resonant gravity waves, where the distance of the arrowhead from this curve quantifies the degree of non-resonance.

as much by the available modal distribution as it is by the structure of the interaction coefficients.

\subsection{Idealized solution: single potential-vorticity and gravity mode}

To compare the predictions of resonant triad theory to solutions of the shallow-water equations, we now consider an idealized system whose initial condition consists of a single gravity mode along $\boldsymbol{k}_{3}$ of amplitude $p_{3}$ propagating through a single PV mode along $\boldsymbol{k}_{2}$ of amplitude $q_{2}$. Since the flow is real-valued, the conjugate PV mode $-\boldsymbol{k}_{2}$ of amplitude $q_{2}^{*}$ will also be present. The configuration is illustrated in figure 2 . The PV mode $\boldsymbol{k}_{2}$ is chosen so that the forced gravity mode $\boldsymbol{k}_{1}=\boldsymbol{k}_{2}+\boldsymbol{k}_{3}$ has a wavelength equal to $\boldsymbol{k}_{3}$ and forms a resonant triad. If we assume that this exchange is substantial, then there will also be a converse scattering of energy back to $\boldsymbol{k}_{3}$ due to the conjugate PV mode, $-\boldsymbol{k}_{2}$, since $\boldsymbol{k}_{3}=\boldsymbol{k}_{1}-\boldsymbol{k}_{2}$. The non-resonant triads $\boldsymbol{k}_{4}=\boldsymbol{k}_{3}-\boldsymbol{k}_{2}$ and $\boldsymbol{k}_{5}=\boldsymbol{k}_{1}+\boldsymbol{k}_{2}$ are also considered, so that we may compare the behaviour of resonant and non-resonant interactions. Similar non-resonant transfers to PV modes associated with these wavenumbers are also present.

If the PV and gravity wave amplitudes for a mode $\boldsymbol{k}_{j}$ are denoted by $q_{j}$ and $p_{j}$, respectively, then the resonant triad equations for each mode are

$$
\begin{aligned}
& \frac{\partial p_{1}}{\partial \tau}=2 \Gamma_{123}^{+0+} q_{2} p_{3}, \\
& \frac{\partial q_{2}}{\partial \tau}=0, \\
& \frac{\partial p_{3}}{\partial \tau}=2 \Gamma_{3-21}^{+0+} q_{2}^{*} p_{1} .
\end{aligned}
$$

The factor of two appears because the triads $\left(\boldsymbol{k}_{1}, \boldsymbol{k}_{2}, \boldsymbol{k}_{3}\right)$ and $\left(\boldsymbol{k}_{1}, \boldsymbol{k}_{3}, \boldsymbol{k}_{2}\right)$ are treated independently in the integration of (2.14). The first equation of (3.11) describes the 
resonant forcing of the $\boldsymbol{k}_{2} \mathrm{PV}$ mode and the $\boldsymbol{k}_{3}$ gravity mode onto the $\boldsymbol{k}_{1}$ gravity mode. The second equation emphasizes the absence of any resonant forcing on the PV mode. The third equation represents the converse scattering of energy from $\boldsymbol{k}_{1}$ to $\boldsymbol{k}_{3}$. Because $q_{2}$ evolves independently of the gravity wave field, the system remains linear and the gravity wave amplitudes $p_{1}$ and $p_{3}$ satisfy the equation

$$
\frac{\partial^{2} p_{j}}{\partial \tau^{2}}+4\left|\Gamma_{123}^{+0+}\right|^{2}\left|q_{2}\right|^{2} p_{j}=0 .
$$

The solutions are sinusoidal oscillations, with a natural frequency $\Omega=2\left|\Gamma_{123}^{+0+}\right|\left|q_{2}\right|$. For the initial conditions $p_{1}(0)=0, p_{3}(0)=A$, the solutions are

$$
\begin{aligned}
& p_{1}=A \mathrm{e}^{\mathrm{i} \varphi} \sin \Omega \tau, \\
& p_{3}=A \cos \Omega \tau,
\end{aligned}
$$

where $\mathrm{e}^{\mathrm{i} \varphi}=-2 \Gamma_{123}^{+0+} q_{2} / \Omega$. After an interval $\Delta t=\pi / 2 \epsilon \Omega$, all of the energy in $\boldsymbol{k}_{3}$ is transferred to $\boldsymbol{k}_{1}$, representing a dominant interaction on an $O\left(\epsilon^{-1}\right)$ time scale due exclusively to resonant wave-PV triads. The non-resonant gravity wave response can be calculated by neglecting any $O\left(\epsilon^{2}\right)$ resonances and by integrating (2.15) with initial condition (2.16), yielding

$$
\begin{aligned}
& p_{4}=\epsilon A \frac{\Gamma_{4-23}^{+0+} q_{2}^{*}}{\mathrm{i}\left(\omega_{4}-\omega_{3}\right)}\left(\mathrm{e}^{-\mathrm{i} \omega_{3} t} \cos \Omega \tau-\mathrm{e}^{-\mathrm{i} \omega_{4} t}\right), \\
& p_{5}=\epsilon A \frac{\Gamma_{521}^{+0+} q_{2}}{\mathrm{i}\left(\omega_{5}-\omega_{1}\right)} \mathrm{e}^{-\mathrm{i} \omega_{1} t} \sin \Omega \tau .
\end{aligned}
$$

The set of corresponding PV amplitudes $\left\{q_{1}, q_{3}, q_{4}, q_{5}\right\}$ are also non-resonant and satisfy similar expressions. Resonant triad theory predicts that (3.13) and (3.14) will remain accurate for at least $O\left(\epsilon^{-1}\right)$ time intervals, and that all non-resonant responses will remain $O(\epsilon)$ quantities during this time.

Numerical calculations of the modes from the full shallow-water equations are shown in figure 3 with $c=f=1$ and $\epsilon=0.1$. We use the initial condition $q_{2}=p_{3}=0.5$ (so that $A=0.5$ ) with all other modes set to zero, and project the modes onto the physical variables. Then we numerically integrate the shallow-water equations up to $t=1000$ and project the solution onto the relevant eigenmodes, producing a time series for each mode of interest. Each curve corresponds to the mode amplitudes for each of the wavenumbers $\boldsymbol{k}_{j}$ depicted in figure 2. The incident wavenumber is $\boldsymbol{k}_{3}=(K, 0)$, the resonantly scattered wavenumber is $\boldsymbol{k}_{1}=(K \cos \theta, K \sin \theta)$, and the other modes are determined from the corresponding triad constraints. The amplitudes in the figure were calculated for $K=1, \theta=\pi / 2$. Resonant triad solutions for modes $q_{2}, p_{3}, p_{4}$ and $p_{5}$ are denoted by thin dotted lines in the figure. (For $p_{4}$, only the envelope over the high-frequency signal is shown.) At early times, there is almost perfect agreement between the analytical and numerical solutions. Modal exchange is dominated by resonant triads, while the non-resonant modes remain as high-frequency $O(\epsilon)$ noise. Significant deviations begin to emerge after $t \approx 400$. The initial PV mode amplitude begins to decrease, in contradiction of the resonant triad assumptions, and ultimately loses almost all of its energy by $t=1000$, which has been transferred to the non-resonant PV modes $q_{1}$ and $q_{3}$. The pair of resonant gravity modes lose their energy as well (to the $-\boldsymbol{k}_{1}$ and $-\boldsymbol{k}_{3}$ modes, not shown in the figure). The nonresonant gravity waves $\left|p_{4}\right|$ and $\left|p_{5}\right|$ deviate strongly from the theoretical solutions, but they remain low-amplitude noise. However, despite the complete breakdown of 
(a)

Resonant amplitude evolution for $K=1, \theta=\pi / 2$

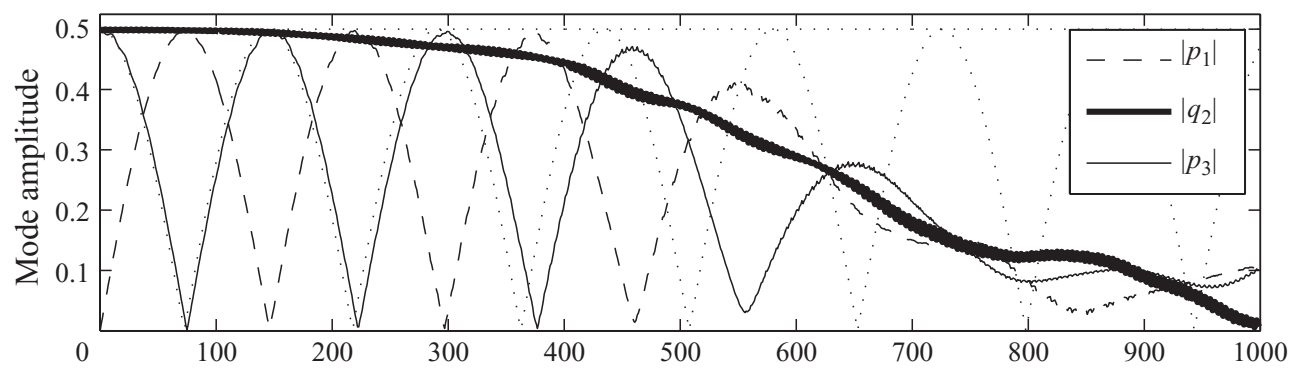

(b)

Non-resonant gravity wave response

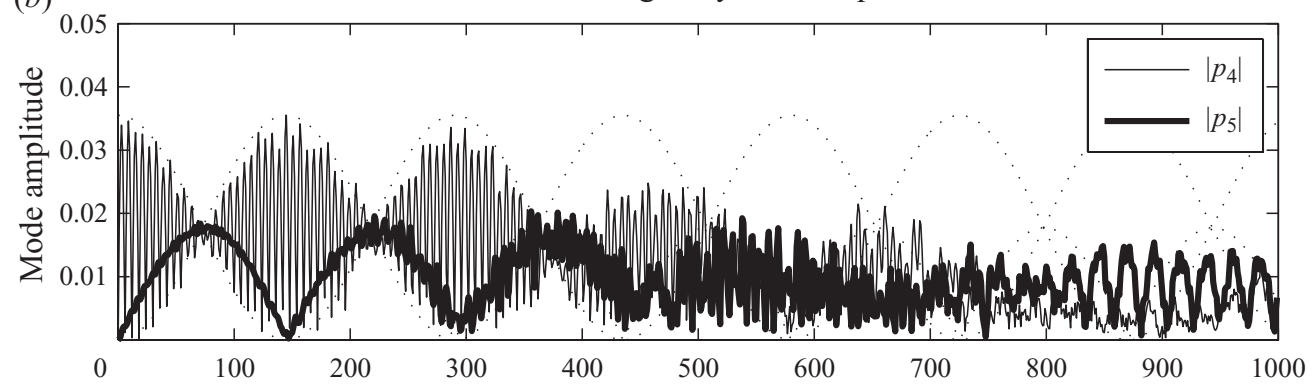

(c)

Non-resonant PV response

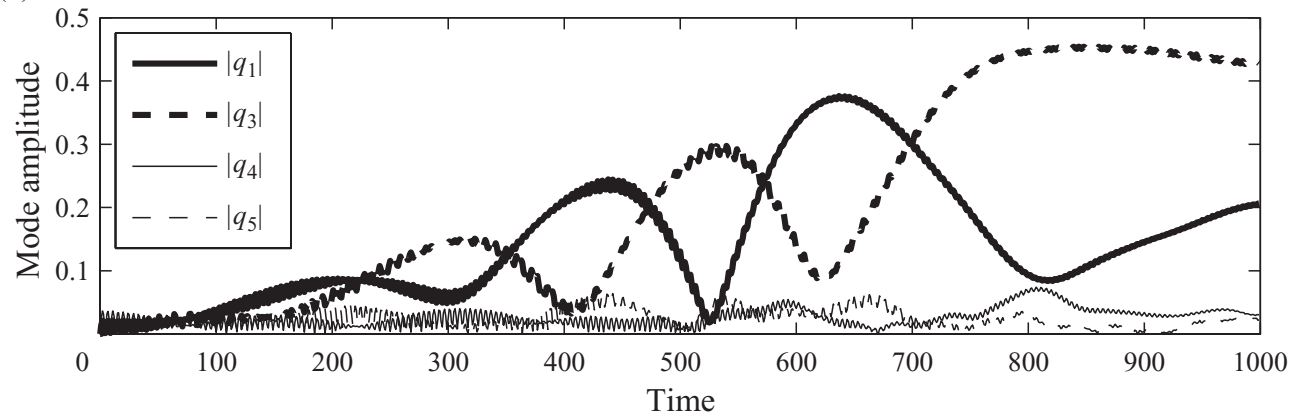

FiguRE 3. Numerical solution of the mode amplitudes for the system proposed in $\S 3.4$ and illustrated in figure 2. Only the modes depicted in figure 2 are shown. Thin dotted lines correspond to theoretical resonant triad solutions for $\left|q_{2}\right|,\left|p_{3}\right|,\left|p_{4}\right|$ (for the low-frequency envelope) and $\left|p_{5}\right|$.

the resonant triad solution, there is no net exchange of energy between the PV and gravity wave modes, and the two fields remain energetically isolated.

Over $O\left(\epsilon^{-1}\right)$ times, the theoretical solutions remain accurate and resonant triad theory should remain applicable if we restrict ourselves to a suitably chosen time interval. To assess the accuracy more thoroughly, we compare the resonant triad and numerical solutions for a number of triads across the interval $\Delta t=400$. Theoretical and numerical calculations of the exchange frequencies $\Omega$ are tested over a range of wavelengths $K$ and angles $\theta$ in figure 4. The lines are the theoretical expressions for $\Omega(K, \theta)$, while data points correspond to least squares estimates of $\Omega$ from numerical solutions. The plots show that there is strong agreement between theoretical and numerical calculations for moderate wavenumbers. Deviations appear as $K$ 


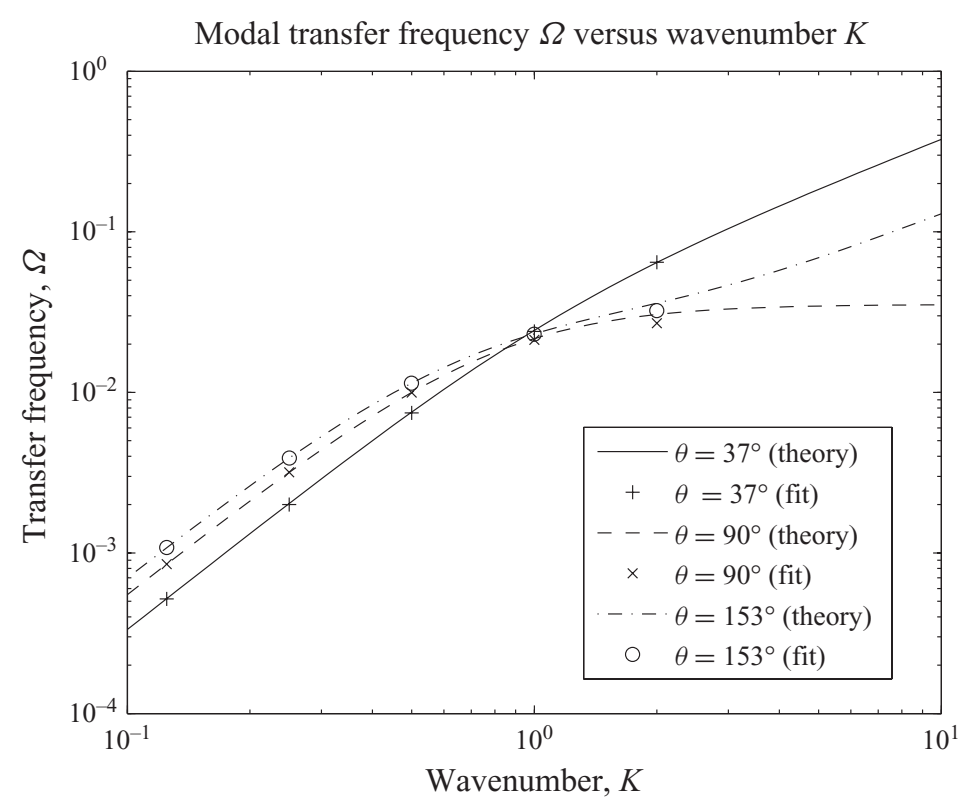

FIGURE 4. Theoretically predicted single-mode exchange frequencies $\Omega(K, \theta)$, shown as curves, are compared to least-squares estimates of $\Omega$ for three representative angles in the numerical primitive equation model.

increases, as the assumption of weak nonlinearity becomes violated and non-resonant interactions emerge, such as observed in figure 3.

These results confirm that PV modes can induce a transfer of energy between gravity waves in a controlled environment. Over $O\left(\epsilon^{-1}\right)$ time intervals, a complete transfer of energy to an otherwise inert gravity mode is possible. Additionally, this interaction is consistent with resonant triad theory, with excellent agreement between theoretical and numerical experiment over early times. Although these results are encouraging, these triads were artificially constructed to be resonant and may not be reflective of more realistic configurations. We attempt to address this issue in $\S 4$.

\section{Gravity waves in a turbulent background}

Using the ideas from $\S 3$, we now attempt to characterize the behaviour of a tidally forced gravity wave as it propagates through an oceanic eddy field. We model this by placing a single monochromatic gravity wave in a turbulent shallow-water PV flow. The turbulent background is generated by initializing the model with a barotropically unstable PV field $q_{I}$ with a spatial distribution

$$
q_{I}(\boldsymbol{x})=q_{0} \cos k_{0} x \cos k_{0} y+\tilde{q}
$$

where $q_{0}$ is a measure of the flow intensity, $k_{0}^{-1}$ characterizes the initial lateral shear, and $\tilde{q}$ is a weak random distribution that destabilizes the flow. For a particular value of $q_{0}$ (between 0.25 and 1.0), the field is initialized using $k_{0}=2$ and uniformly distributed random values between $\pm 10^{-2}$ assigned to $\tilde{q}$ at each point. The PV field is integrated over a time interval of $\Delta t=4000$, producing a characteristically turbulent eddy field that is assumed to be in a state of quasi-equilibrium. A representative snapshot of the height displacement $h$ of the PV field and the corresponding spectral map of the PV mode amplitudes $q_{k}$ are shown in figure 5. For all initial distributions 

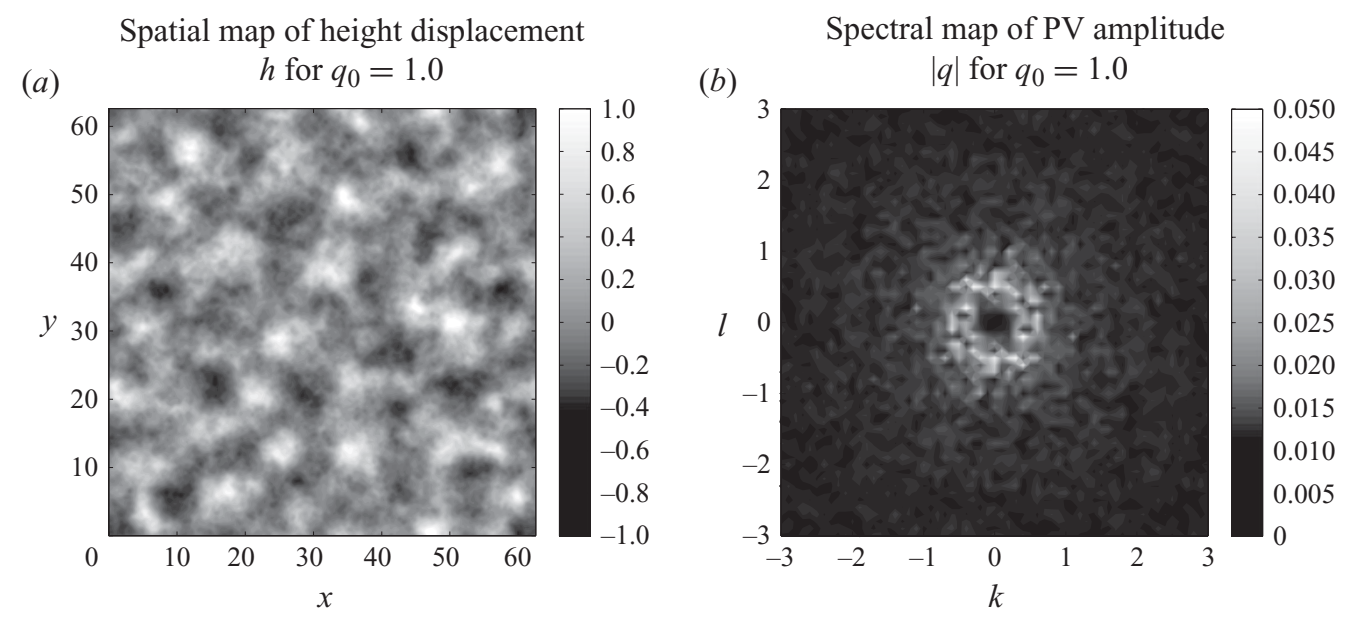

FIGURE 5. Snapshots of the spatial interface displacement $h(\boldsymbol{x})$ and spectral PV mode amplitudes $q_{k}$ for the turbulent background PV field generated with $q_{0}=1.0$ in equation (4.1).
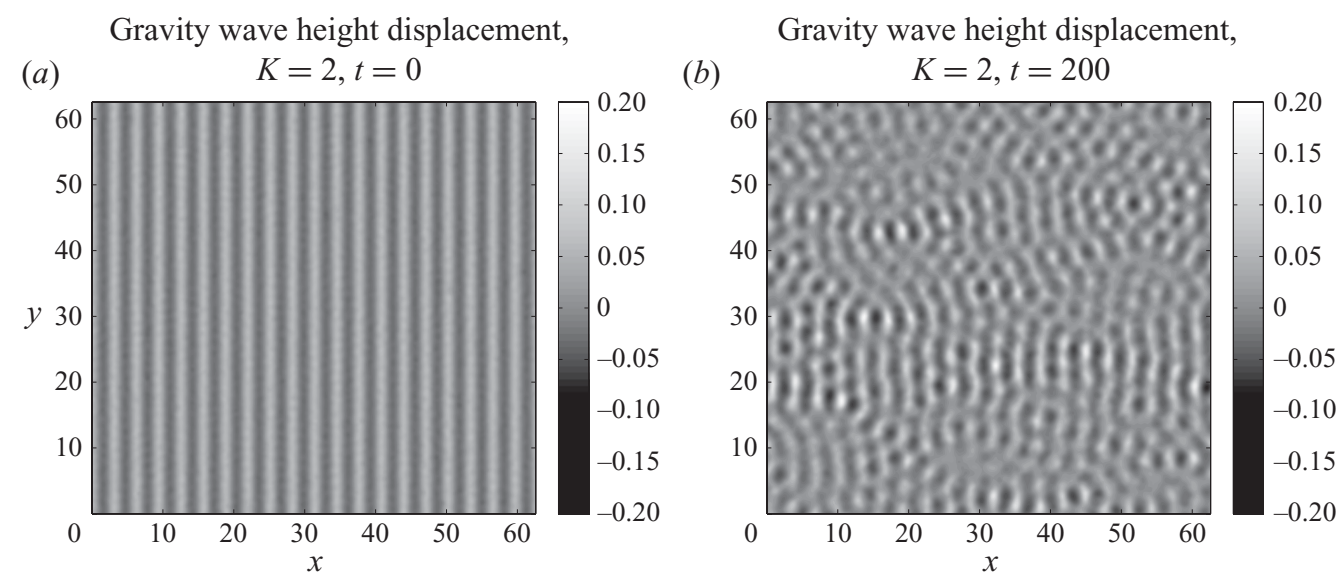

FIGURE 6. The initial and final height displacements for a gravity wave placed in the turbulent PV background after a time interval of $t=200$. Scattering of energy into other modes creates the interference patterns shown on the right.

considered in this paper, the PV field tends to organize itself into eddies of sizes comparable to the deformation radius. This produces a reasonably isotropic spectrum of modes with wavenumbers $|\boldsymbol{k}| \approx 1$, corresponding to the ring in a spectral PV map. After the turbulent flow has been established, we place a single gravity wave of amplitude $p_{0}=0.1$ and a particular mode $K$ onto this field by summation of the wave and PV fields. The model is then integrated over a time interval $\Delta t=200$ to determine the impact on gravity wave propagation.

A snapshot of the gravity wave before and after propagation through the PV field is shown in figure 6, after the PV modes have been removed. Figure $6(b)$ illustrates the distortion of a $K=2$ gravity wave as it propagates through a turbulent background generated from $q_{0}=1$. The ribbon-like bands that appear are the result of interference patterns between the original mode and propagation of scattered energy in different directions. The rate of (linearized) energy loss from the initial 


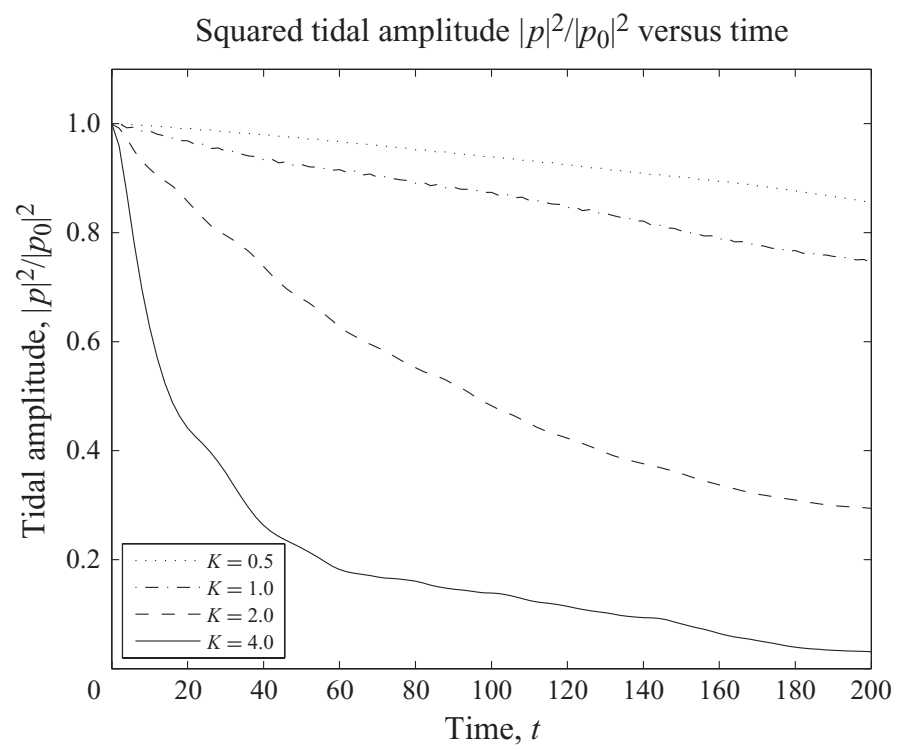

FIGURE 7. Loss of (linearized) energy from the initial gravity wave during propagation through the turbulent PV field. Smaller wavelengths are more sensitive to wave-PV interactions, resulting in a greater loss of energy.

mode in a PV background associated with $q_{0}=1$ is shown in figure 7 . Each curve represents the relative energy contained in the original gravity wave mode within a bandwidth of $\Delta k=0.1$. Large-scale waves in the near-inertial limit retain their energy during propagation through the PV field, even over hundreds of inertial cycles, and essentially preserve their coherent structure. However, the rate of energy loss rapidly increases as the wavelengths become smaller, reflecting the larger values of $\Gamma$ and the stronger nonlinear interaction as $K$ increases. For the waves shown in figure 7, a majority of the energy is lost to scattering after $t>100$ for wavenumbers $K \geqslant 2$.

Although the loss of energy from the initial gravity mode depends on a broad range of processes, it is helpful to assign a single time scale to the energy loss so that we may characterize the decay rate of wave energy over a range of wavenumbers and background PV strengths. We attempt to characterize the energy loss rate of each run by comparing it to an exponential decay of the form

$$
\frac{1}{2}\left|p_{k}(t)\right|^{2}=\frac{1}{2}\left|p_{0}\right|^{2} \mathrm{e}^{-\kappa t}
$$

where $p_{0}$ is the initial mode amplitude and $\kappa$ is estimated by least squares regression. The decay rate time scales $\kappa^{-1}$ are shown in figure 8 . We can immediately see that the rate of energy loss becomes greater as the PV background strength is increased for all wavenumbers $K$, confirming that the PV field is responsible for the energy transfer. The rate of exchange also becomes faster as the wavenumber increases, due to the stronger interaction coefficients at large $K$. There is weaker variation for very high wavenumbers, which we attribute to a saturation effect from an early rapid loss of energy, which is also visible in figure 7.

While the importance of PV in gravity wave scattering is established in figure 8 , a more detailed look at the mode distribution reveals that it is also consistent with the single-triad results in $\S 3$. Figure 9 shows the resulting modal energy distribution after 


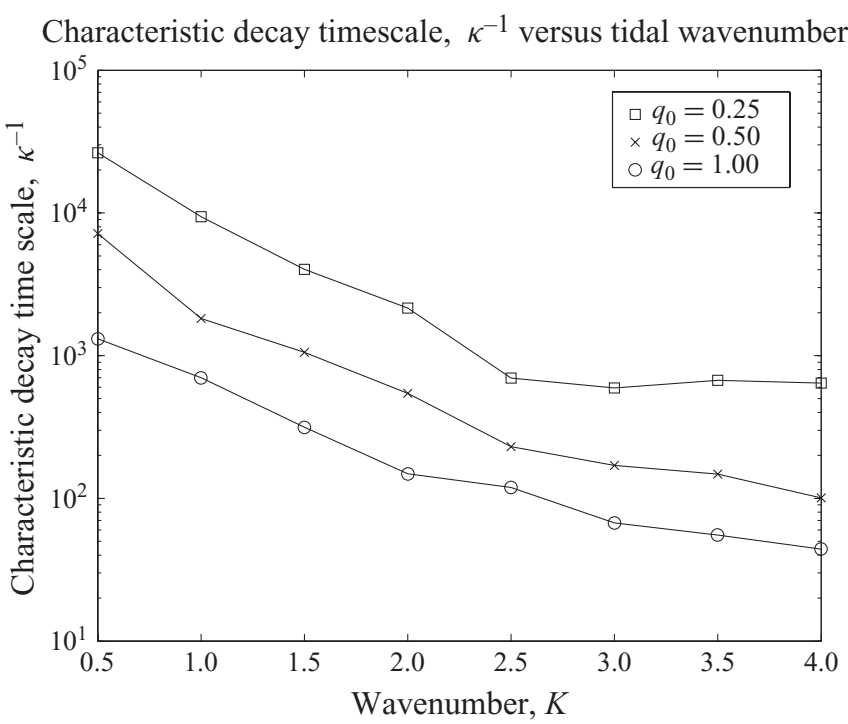

FIGURE 8. Estimates for the time scale for energy loss due to scattering. The rate of energy loss scales consistently with the strength of the background PV field, where shorter time scales correspond to stronger background PV flows.

the gravity waves have propagated in the PV field after a time interval $\Delta t=200$. In every case, the energy in the initial mode $\boldsymbol{k}_{i}=(K, 0)$ is only scattered to other modes of equal wavelength. The absence of substantial waves with different wavelengths confirms that a description based on resonant triads is valid over the parameter regimes presented here, and that both non-resonant and near-resonant interactions play a secondary role in the evolution of the wave spectrum. This result remains robust over very large time intervals (data not shown), eventually producing a fully isotropic spectrum. We also note that there is a subtle radial spread of wave energy for higher values of $K$, representing exchanges to near-resonant modes. These exchanges can become more significant over time and can further detune an initially coherent wave signal. An extreme example of near-resonance is shown in figure 10 for the $K=4$ gravity mode. The transfer of energy to this outer ring is caused by the near-resonant self-interaction of the gravity waves to twice their initial wavenumber, as described in $\$ 3.2$. Although the amount of energy in this outer ring is too small to substantially affect the results in this section, it highlights the emerging influence of near-resonant and off-resonant interactions, which can ultimately dominate the system in the small wavelength limit $(K \gg c / f)$.

The results in $\S 4$ show that the gravity wave field is subject to changes induced by the PV field over time scales that are larger than those associated with wave propagation, but comparable to inertially driven PV flows. The rate of energy loss from the original mode also scales with the strength of the PV background flow, becoming faster as the background becomes stronger. As with the idealized examples of $\S 3$, the wave-PV interaction is dominated by resonant triads. More importantly, it remains a robust feature in these more complex flows. Even though there is a broad isotropic spectrum of PV modes available for the gravity waves to interact with, they consistently select the modes that form resonant triads, producing the ring-like spectral maps shown in figure 9. This interaction becomes more intense as wavenumber $K$ increases, as does the amount of spread to near-resonant modes. But 
(a)

$K=0.5, q_{0}=1$, numerical gravity

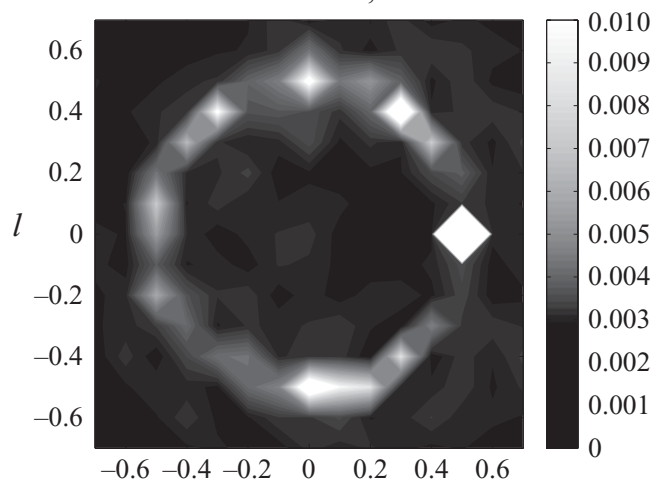

(c) $K=2, q_{0}=1$, numerical gravity

(c) mode distribution, $t=200$

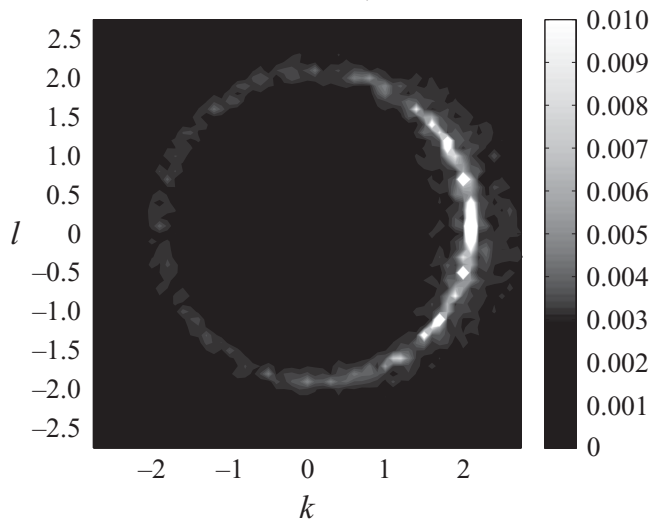

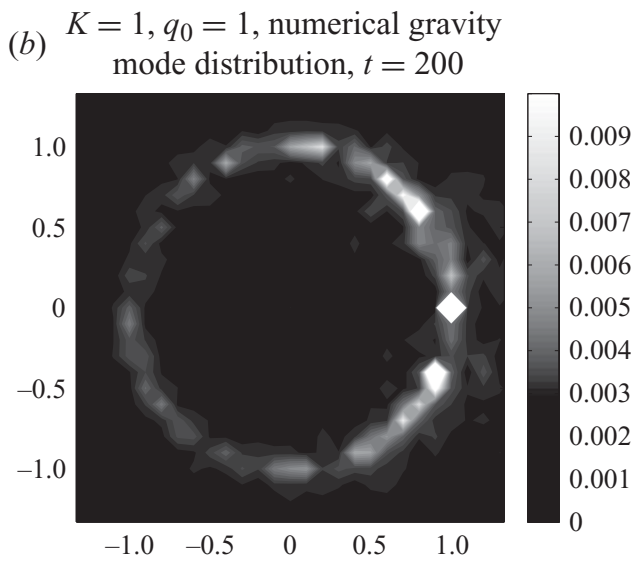

(d) $K=4, q_{0}=1$, numerical gravity

mode distribution, $t=200$

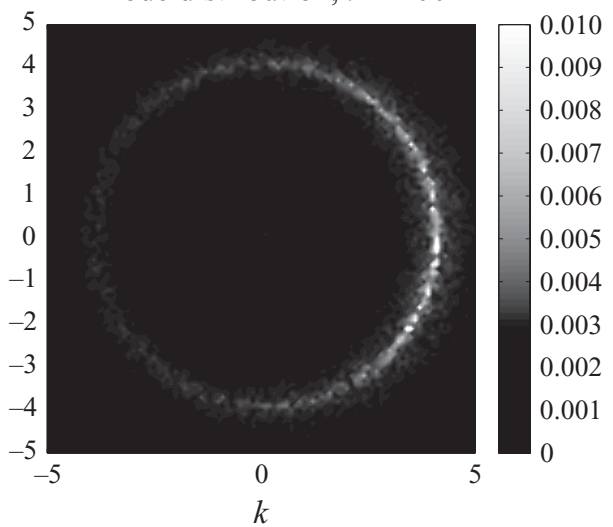

FIGURE 9. Spectral distributions of gravity wave energy of different wavelengths in a turbulent PV background after a time interval $t=200$. The initial energy, concentrated at $\boldsymbol{k}=(K, 0)$, is only scattered to resonant modes of equal wavelength. For larger $K$, there is a radial spread to near-resonant triads.

regardless of the details, there is always a sizeable loss of energy from the initial gravity wave, and we should expect that any coherent gravity wave propagating through a turbulent PV background is unlikely to retain this coherence over inertial times.

\section{Discussion}

\subsection{Scaling to oceanographic conditions}

We now attempt to relate these results to the propagation of an internal tide. We first consider appropriate wavenumbers for the tidally forced spectrum. The dominant contribution to tidal forcing is from the $M_{2}$ mode, with frequency $\omega_{T} \approx 1.4 \times 10^{-4} \mathrm{~s}^{-1}$ (Doodson 1922). If we restrict ourselves to the $M_{2}$ tide, then forcing frequency $\omega_{T}$ has a single fixed value. Since times are scaled relative to $f=2 \Omega_{E} \sin \theta$ for $\Omega_{E}=7.3 \times 10^{-5} \mathrm{~s}^{-1}$ and a latitude $\theta$, we find that the non-dimensional wavenumber 


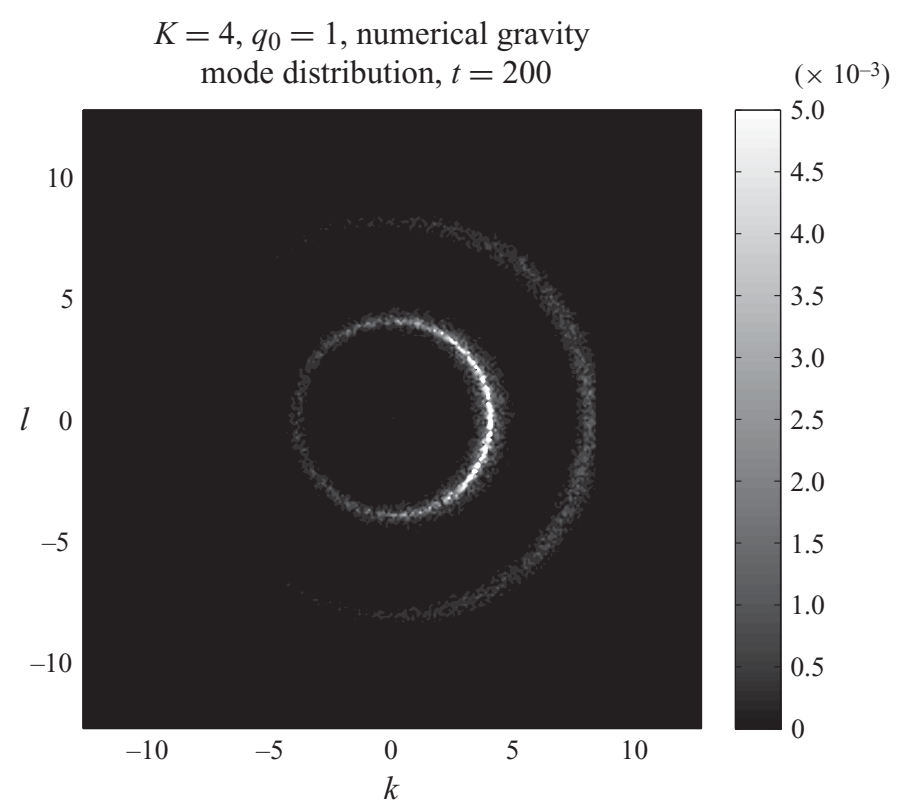

Figure 10. An expanded view of the $K=4$ response spectrum in figure 9. The outer ring of wave energy is due to near-resonant self-interaction of the gravity wave, followed by wave PV scattering along the ring.

$K$ is determined by the latitude of the applied forcing, so that

$$
K=\sqrt{\left(\frac{\omega_{T}}{2 \Omega_{E} \sin \theta}\right)^{2}-1}
$$

A survey of internal tide generation sites by Simmons et al. (2004) shows that the regions of strongest tidal forcing tend to be located within a latitude band of $|\theta|<30^{\circ}$ with corresponding non-dimensional wavenumbers of $K>1.6$. This regime also closely corresponds to the threshold for pronounced PV-induced scattering in our numerical solutions.

If, for the sake of comparison, we accept a shallow-water description and apply our results to the first baroclinic mode, then the mode amplitudes can be related approximately to thermocline displacement, $h$, which in our equations has been scaled relative to $\epsilon$ and the total thermocline depth $H_{0}$. Since we implicitly assume that $|\boldsymbol{u}|$ and $h$ are comparable in magnitude, it is sufficient to relate $q$ or $p$ (the PV and gravity wave amplitudes, respectively) to $h$ for modes where $K$ remains $O(1)$, so that for an $\epsilon$ of 0.1 , PV amplitudes of $q_{0}=1.0$ and gravity wave amplitudes of $p_{0}=0.1$ correspond roughly to $10 \%$ and $1 \%$ displacements of the thermocline, respectively. While a complete description of internal tide dynamics requires a proper representation of the vertical-mode structure and the interactions within, our shallowwater model results represent a valid subset of the complete dynamics of internal wave propagation, and reveals a potential mechanism for the detuning and decoherence of an initially coherent wave, regardless of the vertical-mode structure. The inclusion of vertical-mode interactions may introduce additional mechanisms of tidal decoherence, and further reinforce the importance of the PV field in tidal propagation. 
As for the relative intensity of this mechanism, modes in the vicinity of $K=2$ lose approximately half of their energy over a time interval of $f \Delta t \approx 100$, which corresponds to about 18 days after scaling for the appropriate latitude $\left(\theta \approx 25^{\circ}\right)$; dissipation time scales become even smaller at shorter length scales (or, equivalently, lower latitudes). For a random-phase Garrett-Munk background gravity wave spectrum (Garrett \& Munk 1975) at a similar latitude (30), Olbers \& Pomphrey (1981) estimate that a low baroclinic mode of similar frequency would require approximately 150 days to lose the same amount of energy to higher vertical modes through canonical wave-wave interactions such as PSI (McComas \& Bretherton 1977). We would therefore expect our estimates for wave-PV interactions to be more relevant to coherent tides than those based on tradition statistical treatments summarized in Müller et al. (1986).

For the PSI triad interactions for coherent tides proposed by MacKinnon \& Winters (2005), their estimated dissipation time scales are of the order of 10 days, if not shorter, in the vicinity of the critical $29^{\circ}$ latitude, suggesting that it is the dominant process at this latitude. However, their simulations also show that this mechanism is a relatively localized one, and that there is little dissipation or loss of energy to higher vertical modes outside of this latitude. In contrast, a PV-induced scattering is less sensitive to latitude and depends only on the gravity wavenumber and the local PV field. As a potential detuning mechanism for internal tides, PV-induced scattering may be applicable during propagation between generation and dissipation at $29^{\circ}$, and may lose their coherence as they propagate towards the $29^{\circ}$ latitude.

To explore this possibility, we attempt to assess the impact of wave-PV interaction on the life cycle of the internal tide from our numerical results in $\S 4$. For the modes depicted in figure 7, we assign a half-life to each mode $K$ by identifying the time interval $\Delta t$ over which its relative energy is reduced by $50 \%$. We then calculate a propagation distance $\Delta x=c_{g} \Delta t$, where $c_{g}=K / \sqrt{1+K^{2}}$ is the group velocity. Finally, we scale this result by the deformation radius $c / f$, where $f$ is determined from the latitude associated with the mode $K$ and $c$ is taken as the first baroclinic mode, which is approximately $c_{1} \approx 3.1 \mathrm{~m} \mathrm{~s}^{-1}$ for tropical latitudes (Rainville \& Pinkel 2006). The resulting propagation distance is reported in degrees of latitude, although propagation can conceivably occur in any direction. The results are shown in figure 11. The estimates show that these waves lose a substantial amount of energy after propagation through approximately $10^{\circ}$ of latitude, with smaller scale waves undergoing more rapid dispersion. While turbulent conditions can vary greatly over the ocean, and crucial parameters such as $f$ will change as the waves propagate through new latitudes, these results show that a vigorous PV field is capable of dispersing internal tides and producing a more disorganized and isotropic response over substantial time scales.

\subsection{Comparison to prior study}

We now attempt to relate our results to prior research on near-inertial wave propagation through a PV field. Despite the different approaches, our results are consistent with the theoretical models of Kunze (1985) and Young \& Ben Jelloul (1997), even though these studies are primarily focused on the vertical structure of waves in the near-inertial limit, $K \ll f / c$. For a fixed vertical mode in a continuously stratified background, the gravity wave dispersion relation reduces to $\omega \approx f$, implying that all $( \pm, 0, \pm)$ triad interactions in the near-inertial regime are effectively resonant, and that the horizontal advection terms are preserved in their entirety. This is reflected 


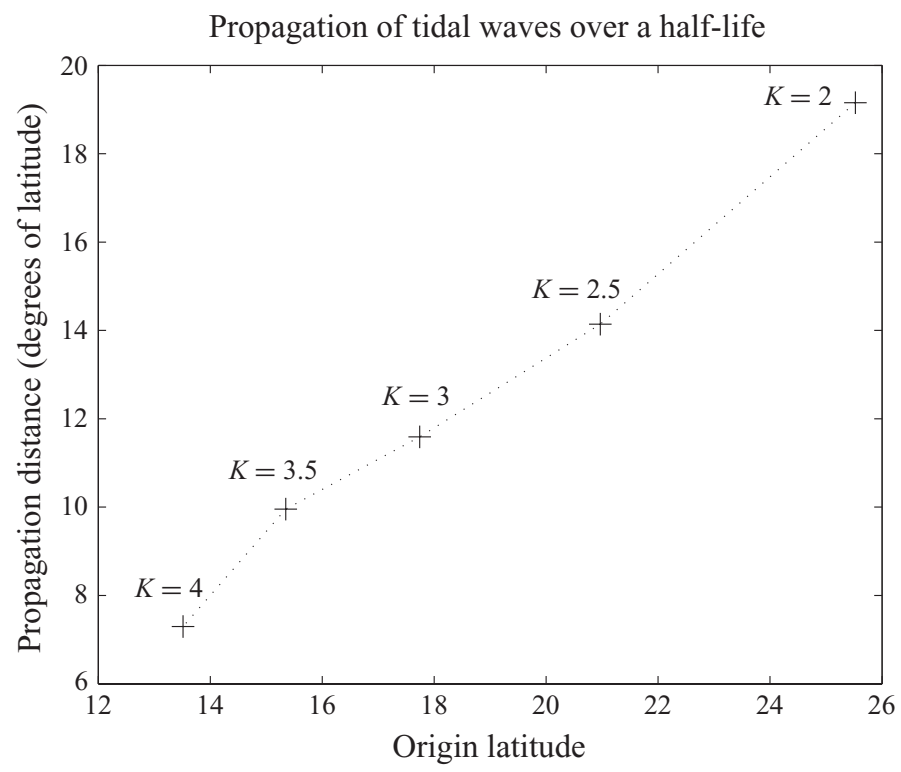

FIGURE 11. The propagation distance of a particular gravity mode $K$ over the effective half-life of the mode during propagation through the turbulent PV field generated by $q_{0}=1.0$ is shown. Each mode is scaled to its corresponding origin latitude, as outlined in the text, and propagation distance has been expressed in degrees of latitude, although propagation can occur in any direction. The half-life is determined from simulation results such as in figure 7.

in the barotropic eikonal equation of Kunze (1985),

$$
\frac{\mathrm{d} \boldsymbol{k}}{\mathrm{d} t}=-\nabla \zeta
$$

where $\zeta$ is the vertical vorticity. This equation resembles the single-triad transfer of energy from mode $\boldsymbol{k}$ to $\boldsymbol{k}+\delta \boldsymbol{k}$ along a PV gradient $\delta \boldsymbol{k}$, such as described $\S 3$. A major difference, however, is that this equation contains no wavelength selection criterion, so that any PV mode is permitted to direct the propagation of the waves, regardless of wavenumber. Similarly, Young \& Ben Jelloul (1997) retain the complete horizontal advection term in their equation (2.24). The horizontal advection is also largely neglected in these studies in favour of vertical-mode interactions, but this is also consistent with our analysis, since we have shown that horizontal interactions are relatively weak in the near-inertial spectrum and only emerge when $K=O(c / f)$.

Our results appear to contradict the conclusions of Reznik et al. (2001), who argue that there can be no persistent interaction between smooth and bounded PV and gravity wave distributions in the asymptotic time limit $t \rightarrow \infty$. However, we note a number of key distinctions between their assumptions and our own. First, our distributions are not spatially bounded, due to the use of purely monochromatic modes in periodic domains, which represents both a theoretical convenience and a numerical necessity. Spectrally, the fields consist of sums of Dirac $\delta$-distributions of energy into specific modes, so that integration over the surface of resonant triads in (2.14) defined by the constraint $\omega_{123}^{i j k}=0$ produces finite values. A continuous distribution, on the other hand, would produce no such contribution, since integration is over a measure-zero set. However, we believe that such issues are only strictly applicable in the asymptotic limit $t \rightarrow \infty$. In the intermediate limit of $t \rightarrow \epsilon^{-1}$, both resonant and 
near-resonant triads can make comparable contributions to the wave field, so that the resonant surface becomes a resonant skin of finite thickness, or $O(\epsilon)$ bandwidth. In this case, the distinction between a $\delta$-distribution, a numerically resolved spectral grid point, and a smoothly varying but narrow peak of $O(\epsilon)$ bandwidth should be of secondary importance in any resonant triad solutions, and our results should remain relevant to the problem of tidal propagation.

The results presented in this paper have consequences for any study that relies on the existence and persistence of coherent gravity waves in the open ocean. The rapid dispersion of coherent internal tides based on the PSI mechanism (MacKinnon \& Winters 2005) may be sensitive to the effects of PV-induced forcing, as would the projected trajectories of internal waves in the open ocean (Rainville \& Pinkel 2006). The scattering of energy in other directions could greatly reduce the amplitude of any waves that reach the critical $29^{\circ}$ latitude. Prolonged exposure to geostrophic turbulence would also tend to randomize the phases of any coherent wavepackets, which could further reduce the effectiveness of these proposed dissipation mechanisms. $\mathrm{PV}$-induced scattering could even lead to a statistical homogenization of the wave spectrum. For example, observational evidence of a suppressed PSI instability and a broadening and detuning of internal waves (Alford et al. 2007) may be related to a PV-induced scattering of wave energy.

\section{Summary}

On geostrophic length scales and inertial time scales, PV induces substantial scattering of a gravity wave field in a shallow-water model. Although there is no exchange of energy between the PV field and the gravity waves, the PV acts as a catalyst for the redistribution of energy within the gravity wave spectrum. This interaction between PV and gravity waves is also consistent with predictions by resonant triad theory. Gravity waves can only resonate and transfer energy to other waves of equal wavelength, but in any direction of propagation. A single coherent wavepacket in a PV field will, under most circumstances, tend to fan out and form a broad radiative beam as it propagates through an active geostrophic flow. This will ultimately lead to a more disorganized and incoherent wave spectrum.

The role of resonant triad theory is strongly supported by the close agreement between the full numerical solutions and the theoretically predicted modulational equations for the wave amplitudes. We have illustrated that a gravity wave is capable of transferring all of its energy to another resonant mode, resulting in a sinusoidal exchange at a frequency that depends entirely on the nonlinear terms. The close agreement between the numerical and resonant triad solutions also motivates the possibility of a reduced set of equations that can accurately forecast the evolution of the gravity wave field on the inertial time scale, producing slightly less accurate, but more efficient forecasts on a coarse space and time grid that is more appropriate for global modelling. Progress in this area would require the introduction of new ideas related to near-resonant interactions that are beyond the scope of this paper, but preliminary results are encouraging and offer the possibility of efficient global internal wave modelling.

\section{Appendix A. Normal modes for the shallow-water system}

Evaluation of the modal equations of motion (2.9) and comparison to physical variables requires the calculation of the eigenmodes $\Psi_{k}^{s}$ and interaction coefficients 
$\Gamma_{123}^{i j k}$. To do this, we begin with (2.3) and apply Fourier transforms from $\boldsymbol{x}$ to $\boldsymbol{k}$ of the form $\mathscr{F}[g](\boldsymbol{x})=\left(1 /(2 \pi)^{2}\right) \int g(\boldsymbol{x}) \mathrm{e}^{-\mathrm{i} k \boldsymbol{x}} \mathrm{d} \boldsymbol{x}$. The spectral representation is

$$
\frac{\partial \Phi_{k}}{\partial t}+\mathrm{i} H \Phi_{k}=\epsilon \mathscr{N}_{k}
$$

where $\Phi_{k}=\left(u_{k}, v_{k}, h_{k}\right)^{\mathrm{T}}, H$ is the Hermitian operator

$$
H=\left(\begin{array}{ccc}
0 & \mathrm{i} f & c k \\
-\mathrm{i} f & 0 & c l \\
c k & c l & 0
\end{array}\right)
$$

and $\mathscr{N}_{k}=\left(\mathscr{N}_{k}^{u}, \mathscr{N}_{k}^{v}, \mathscr{N}_{k}^{h}\right)^{\mathrm{T}}$ denotes the nonlinear terms, given by

$$
\begin{aligned}
& \mathscr{N}_{k}^{u}=-\mathrm{i} \int\left(k_{3} u_{2} u_{3}+l_{3} v_{2} u_{3}\right) \delta_{2+3}^{k} \mathrm{~d} \boldsymbol{k}_{23}, \\
& \mathscr{N}_{k}^{v}=-\mathrm{i} \int\left(k_{3} u_{2} v_{3}+l_{3} v_{2} v_{3}\right) \delta_{2+3}^{k} \mathrm{~d} \boldsymbol{k}_{23}, \\
& \mathscr{N}_{k}^{h}=-\mathrm{i} \int\left(\left(k_{2}+k_{3}\right) h_{2} u_{3}+\left(l_{2}+l_{3}\right) h_{2} v_{3}\right) \delta_{2+3}^{k} \mathrm{~d} \boldsymbol{k}_{23} .
\end{aligned}
$$

We have introduced the notation $\delta_{2+3}^{1}=\delta\left(\boldsymbol{k}_{1}-\boldsymbol{k}_{2}-\boldsymbol{k}_{3}\right)$ for the Dirac delta functions, $\mathrm{d} \boldsymbol{k}_{23}=\mathrm{d} \boldsymbol{k}_{2} \mathrm{~d} \boldsymbol{k}_{3}$ for the integral measures and $\phi_{n}=\phi\left(\boldsymbol{k}_{n}\right)$ for $\phi \in\{u, v, h\}$. Unless stated otherwise, all integrations are over the entire domains.

The nonlinear terms can be written succinctly in the form

$$
\mathscr{N}_{1}^{\alpha}=\int_{23} \sum_{\beta \gamma} F_{123}^{\alpha \beta \gamma}\left(\Phi_{2}\right)_{\beta}\left(\Phi_{3}\right)_{\gamma} \mathrm{d} \boldsymbol{k}_{23},
$$

where $\{\alpha, \beta, \gamma\}$ represent indices over physical mode amplitudes $\{u, v, h\}, \Phi_{n}=\Phi\left(\boldsymbol{k}_{n}\right)$ and $\left(\Phi_{k}\right)_{\alpha}$ denotes a particular component of $\Phi_{k}$. The coefficients $F_{123}^{\alpha \beta \gamma}$ are determined by the shallow-water model. We can assume without loss of generality that $F_{123}^{\alpha \beta \gamma}$ has the symmetry

$$
F_{123}^{\alpha \beta \gamma}=F_{132}^{\alpha \gamma \beta},
$$

since the asymmetric component does not contribute to the integral and can be removed. This is a reflection of the arbitrary labelling of $\boldsymbol{k}_{2}$ and $\boldsymbol{k}_{3}$ in the convolution integral. For the shallow-water model, these coefficients are

$$
\begin{array}{lll}
F_{123}^{u u u}=-\frac{\mathrm{i}}{2}\left(k_{2}+k_{3}\right), & F_{123}^{v u v}=-\frac{\mathrm{i}}{2} k_{3}, & F_{123}^{h u h}=-\frac{\mathrm{i}}{2}\left(k_{2}+k_{3}\right), \\
F_{123}^{u u v}=-\frac{\mathrm{i}}{2} l_{2}, & F_{123}^{v v u}=-\frac{\mathrm{i}}{2} k_{2}, & F_{123}^{h v h}=-\frac{\mathrm{i}}{2}\left(l_{2}+l_{3}\right), \\
F_{123}^{u v u}=-\frac{\mathrm{i}}{2} l_{3}, & F_{123}^{v v v}=-\frac{\mathrm{i}}{2}\left(l_{2}+l_{3}\right), & F_{123}^{h h u}=-\frac{\mathrm{i}}{2}\left(k_{2}+k_{3}\right), \\
& & F_{123}^{h h v}=-\frac{\mathrm{i}}{2}\left(l_{2}+l_{3}\right),
\end{array}
$$

where the asymmetric components have been removed. The triad constraint $\delta_{2+3}^{1}$ has been omitted for convenience. All other unlisted coefficients are equal to zero. 
The eigenmodes are determined from the linear operator $H$. Each of the eigenvectors $\Psi_{k}^{s}$ can be compactly written as

$$
\Psi_{\boldsymbol{k}}^{s}=\frac{1}{C_{\boldsymbol{k}}^{s}}\left(\omega_{\boldsymbol{k}}^{s} \Psi_{\boldsymbol{k}}^{\omega}+f \Psi_{\boldsymbol{k}}^{f}\right)
$$

where

$$
\Psi_{k}^{\omega}=\left(\begin{array}{c}
c k \\
c l \\
\omega
\end{array}\right), \quad \Psi_{k}^{f}=\left(\begin{array}{c}
\mathrm{i} c l \\
-\mathrm{i} c k \\
-f
\end{array}\right)
$$

and

$$
C_{k}^{0}=f \Omega_{k}, \quad C_{k}^{ \pm}=\sqrt{2} c K \Omega_{k}
$$

When we write $\Psi_{\boldsymbol{k}}^{\omega}$, it is understood that $\omega$ refers to $\omega_{\boldsymbol{k}}^{s}=s \Omega_{\boldsymbol{k}}$. The first vector in $\Psi_{\boldsymbol{k}}^{s}$ describes the non-dispersive propagation of a non-rotating gravity wave, while the second describes a geostrophically balanced mode.

Since $H$ is Hermitian, the eigenvectors are orthogonal and form a complete basis for solutions to the unbounded shallow-water equations. Any flow can be fully described by either the physical mode amplitudes or the eigenmode amplitudes, which are related to each other by

$$
\Phi_{k}(t)=\sum_{s=0, \pm} A_{k}^{s}(t) \Psi_{k}^{s}, \quad A_{k}^{s}(t)=\left(\Psi_{k}^{s}\right)^{\dagger} \Phi_{k}(t)
$$

where the dagger denotes the Hermitian conjugate. When projected onto the eigenmodes, the equations of motion become

$$
\frac{\partial A_{k}^{s}}{\partial t}+\mathrm{i} \omega_{k}^{s} A_{k}^{s}=\epsilon N_{k}^{s}
$$

If we now expand each element of $\Phi_{k}$ in terms of the eigenmodes, and project the nonlinearity $\mathscr{N}_{\boldsymbol{k}}$ onto a particular component, so that

$$
\left(\Phi_{1}\right)_{\alpha}=\sum_{i} A_{1}^{i}\left(\Psi_{k}^{i}\right)_{\alpha}, \quad N_{k}^{s}=\sum_{\alpha}\left(\Psi_{k}^{s}\right)_{\alpha}^{*} \mathscr{N}_{k}^{\alpha},
$$

where $\left(\Psi_{k}^{i}\right)_{\alpha}$ denotes the $\alpha$ component of $\Psi_{k}^{i}$ and $*$ is the complex conjugate, then the nonlinear components become

$$
N_{\boldsymbol{k}}^{i}=\sum_{j k} \int_{23} \sum_{\alpha \beta \gamma} F_{123}^{\alpha \beta \gamma}\left(\Psi_{1}^{i}\right)_{\alpha}^{*}\left(\Psi_{2}^{j}\right)_{\beta}\left(\Psi_{3}^{k}\right)_{\gamma} A_{2}^{j} A_{3}^{k} \mathrm{~d} \boldsymbol{k}_{23}
$$

This expression can be simplified if we define the 'interaction coefficients' as

$$
\Gamma_{123}^{i j k}=\sum_{\alpha, \beta, \gamma} F_{123}^{\alpha \beta \gamma}\left(\Psi_{1}^{i}\right)_{\alpha}^{*}\left(\Psi_{2}^{j}\right)_{\beta}\left(\Psi_{3}^{k}\right)_{\gamma} .
$$


Since the flow solutions are real-valued, and since the eigenvalues and eigenvectors have the properties

$$
\omega_{-k}^{-s}=-\omega_{k}^{s}, \quad\left(\Psi_{k}^{s}\right)^{*}=\Psi_{-k}^{-s},
$$

the amplitudes and interaction coefficients must satisfy the conditions

$$
\left(A_{k}^{s}\right)^{*}=A_{-k}^{-s}, \quad\left(\Gamma_{123}^{i j k}\right)^{*}=\Gamma_{-1-2-3}^{-i-j-k} .
$$

Additionally, the symmetry of $F_{123}^{\alpha \beta \gamma}$ carries over to the interaction coefficients so that

$$
\Gamma_{123}^{i j k}=\Gamma_{132}^{i k j}
$$

In terms of $\Gamma_{123}^{i j k}$, the eigenmode equations of motion are

$$
\frac{\partial A_{1}^{i}}{\partial t}+\mathrm{i} \omega_{1}^{i} A_{1}^{i}=\epsilon \sum_{j k} \int_{23} \Gamma_{123}^{i j k} A_{2}^{j} A_{3}^{k} \mathrm{~d} \boldsymbol{k}_{23}
$$

These are the equations that we will use in this study and represent a complete description of the flow in terms of the eigenmodes.

Using our decomposition of $\Psi_{k}^{s}$, it is possible to express $\Gamma_{123}^{i j k}$ as a sequence of independent elements describing the interaction between non-dispersive gravity waves $\Psi_{k}^{\omega}$ and geostrophically balanced modes $\Psi_{k}^{f}$. Under this representation, the interaction coefficients are

$$
\begin{aligned}
\Gamma_{123}^{s_{1} s_{2} s_{3}}= & \frac{1}{C_{1}^{s_{1}} C_{2}^{s_{2}} C_{3}^{s_{3}}}\left\{f^{3} \Gamma_{123}^{f f f}+f^{2} \omega_{1} \Gamma_{123}^{\omega f f}+f^{2} \omega_{2} \Gamma_{123}^{f \omega f}+f^{2} \omega_{3} \Gamma_{123}^{f f \omega}\right. \\
& \left.+f \omega_{1} \omega_{2} \Gamma_{123}^{f \omega \omega}+f \omega_{1} \omega_{3} \Gamma_{123}^{f f \omega}+f \omega_{2} \omega_{3} \Gamma_{123}^{f \omega \omega}+\omega_{1} \omega_{2} \omega_{3} \Gamma_{123}^{\omega \omega \omega}\right\},
\end{aligned}
$$

where

$$
\begin{aligned}
\Gamma_{123}^{f f f} & =\frac{c}{2}\left[\boldsymbol{k}_{2} \times \boldsymbol{k}_{3}\right]\left(\Omega_{2}^{2}-\Omega_{3}^{2}\right), \\
\Gamma_{123}^{f f \omega} & =\frac{c}{2}\left[-\mathrm{i} \Omega_{2}^{2}\left(\boldsymbol{k}_{1} \cdot \boldsymbol{k}_{3}\right)+f \omega_{3}\left[\boldsymbol{k}_{2} \times \boldsymbol{k}_{3}\right]\right], \\
\Gamma_{123}^{f \omega \omega} & =\frac{c}{2}\left[\left(\boldsymbol{k}_{1} \cdot \boldsymbol{k}_{3}\right) \mathrm{i} f \omega_{2}+\left(\boldsymbol{k}_{1} \cdot \boldsymbol{k}_{2}\right) \mathrm{i} f \omega_{3}\right], \\
\Gamma_{123}^{\omega f f} & =-\mathrm{i} c^{3}\left[\boldsymbol{k}_{2} \times \boldsymbol{k}_{3}\right]^{2}, \\
\Gamma_{123}^{\omega f \omega} & =-\frac{c}{2}\left[\left(\Omega_{1}^{2}-\Omega_{2}^{2}+\omega_{1} \omega_{3}\right)\left[\boldsymbol{k}_{2} \times \boldsymbol{k}_{3}\right]-\mathrm{i} f \omega_{1}\left(\boldsymbol{k}_{1} \cdot \boldsymbol{k}_{3}\right)\right], \\
\Gamma_{123}^{\omega \omega \omega} & =-\mathrm{i} \frac{c}{2}\left[K_{1}^{2} c^{2}\left(\boldsymbol{k}_{2} \cdot \boldsymbol{k}_{3}\right)+\omega_{1} \omega_{2}\left(\boldsymbol{k}_{1} \cdot \boldsymbol{k}_{3}\right)+\omega_{1} \omega_{3}\left(\boldsymbol{k}_{1} \cdot \boldsymbol{k}_{2}\right)\right] .
\end{aligned}
$$

We have used the shorthand notation $\left[\boldsymbol{k}_{i} \times \boldsymbol{k}_{j}\right] \equiv k_{i} l_{j}-k_{j} l_{i}$. 
Using these expressions, the shallow-water interaction coefficients for each triad type are

$$
\begin{aligned}
\Gamma_{123}^{000}= & \frac{c\left[\boldsymbol{k}_{2} \times \boldsymbol{k}_{3}\right]\left(\Omega_{2}^{2}-\Omega_{3}^{2}\right)}{2\left|\Omega_{1}\right|\left|\Omega_{2}\right|\left|\Omega_{3}\right|}, \\
\Gamma_{123}^{00+}= & \frac{\Omega_{2}^{2}\left(f\left[\boldsymbol{k}_{2} \times \boldsymbol{k}_{3}\right]-\mathrm{i} \Omega_{3}\left(\boldsymbol{k}_{1} \cdot \boldsymbol{k}_{3}\right)\right)}{2 \sqrt{2} K_{3}\left|\Omega_{1}\right|\left|\Omega_{2}\right|\left|\Omega_{3}\right|}, \\
\Gamma_{123}^{0++}= & 0, \\
\Gamma_{123}^{+00}= & \frac{\left[\boldsymbol{k}_{2} \times \boldsymbol{k}_{3}\right]\left(f\left(\Omega_{2}^{2}-\Omega_{3}^{2}\right)-2 \mathrm{i} c^{2} \Omega_{1}\left[\boldsymbol{k}_{2} \times \boldsymbol{k}_{3}\right]\right)}{2 \sqrt{2} K_{1}\left|\Omega_{1}\right|\left|\Omega_{2}\right|\left|\Omega_{3}\right|} \\
\Gamma_{123}^{+0+}= & \left\{\left[\boldsymbol{k}_{2} \times \boldsymbol{k}_{3}\right]\left(\left(f^{2}+\Omega_{1} \Omega_{3}\right) \Omega_{2}^{2}-\Omega_{1}^{2} \Omega_{3}\left(\Omega_{1}+\Omega_{3}\right)\right)\right. \\
& \left.-\mathrm{i} f\left(2 \Omega_{1} c^{2}\left[\boldsymbol{k}_{2} \times \boldsymbol{k}_{3}\right]^{2}+\Omega_{3}\left(\Omega_{2}^{2}-\Omega_{1}^{2}\right)\left(\boldsymbol{k}_{1} \cdot \boldsymbol{k}_{3}\right)\right)\right\} / 4 c K_{1} K_{3}\left|\Omega_{1}\right|\left|\Omega_{2} \| \Omega_{3}\right|, \\
\Gamma_{123}^{+++}= & \left\{f\left[\boldsymbol{k}_{2} \times \boldsymbol{k}_{3}\right] \Omega_{1}\left(\Omega_{1}\left(\Omega_{2}^{2}-\Omega_{3}^{2}\right)+\Omega_{2}\left(\Omega_{1}^{2}-\Omega_{3}^{2}\right)-\Omega_{3}\left(\Omega_{1}^{2}-\Omega_{2}^{2}\right)\right)\right. \\
& -\mathrm{i} \Omega_{1}\left(\left(c^{2} K_{1}^{2}\right) \Omega_{2} \Omega_{3}\left(\boldsymbol{k}_{2} \cdot \boldsymbol{k}_{3}\right)+\left(c^{2} K_{2}^{2}\right) \Omega_{1} \Omega_{3}\left(\boldsymbol{k}_{1} \cdot \boldsymbol{k}_{3}\right)+\left(c^{2} K_{3}^{2}\right) \Omega_{1} \Omega_{2}\right. \\
& \left.\left.\times\left(\boldsymbol{k}_{1} \cdot \boldsymbol{k}_{2}\right)\right)-2 \mathrm{i} \Omega_{1} f^{2} c^{2}\left[\boldsymbol{k}_{2} \times \boldsymbol{k}_{3}\right]^{2}\right\} / 4 \sqrt{2} c^{2} K_{1} K_{2} K_{3}\left|\Omega_{1} \| \Omega_{2}\right|\left|\Omega_{3}\right| .
\end{aligned}
$$

As with $F_{123}^{\alpha \beta \gamma}$, the triad constraint $\delta_{2+3}^{1}$ has been removed from $\Gamma_{123}^{i j k}$ for convenience. Interaction coefficients associated with negative gravity modes $\omega_{k}^{-}$are inferred by replacing frequencies $\Omega_{k}$ of positive gravity wave modes $k$ with $-\Omega_{k}$.

\section{Appendix B. Derivation of (3.3)}

We begin with the PV modulational equation, (3.2),

$$
\frac{\partial a_{1}^{0}}{\partial \tau}=\int_{23} \Gamma_{123}^{000} a_{2}^{0} a_{3}^{0} \mathrm{~d} \boldsymbol{k}_{23} .
$$

The PV mode amplitude $a_{k}^{0}$ is related to geostrophically balanced height $\bar{h}$ by the relation $\bar{h}_{k}=-f a_{k}^{0} / \Omega_{k}$, based on the eigenvector (A 10) for $s=0$. The interaction coefficient $\Gamma_{123}^{000}$ is calculated in Appendix A. Substitution yields

$$
\frac{\partial}{\partial \tau}\left(-\Omega_{1}^{2} \bar{h}_{1}\right)=\frac{c^{3}}{f} \int_{23} \frac{1}{2}\left[\boldsymbol{k}_{2} \times \boldsymbol{k}_{3}\right]\left(K_{2}^{2}-K_{3}^{2}\right) \bar{h}_{2} \bar{h}_{3} \delta_{2+3}^{1} \mathrm{~d} \boldsymbol{k}_{23} .
$$

The last step requires us to identify the right-hand side as the Fourier transform of the Jacobian, $J\left(h, \nabla^{2} h\right)$ :

$$
\begin{aligned}
\mathscr{F}_{\boldsymbol{k}}\left\{J\left(\bar{h}, \nabla^{2} \bar{h}\right)\right\} & =\int_{23}\left(k_{2} l_{3}-k_{3} l_{2}\right) K_{3}^{2} \bar{h}_{2} \bar{h}_{3} \delta_{2+3}^{\boldsymbol{k}} \mathrm{d} \boldsymbol{k}_{23} \\
& =\int_{23} \frac{1}{2}\left[\boldsymbol{k}_{2} \times \boldsymbol{k}_{3}\right]\left(K_{3}^{2}-K_{2}^{2}\right) \bar{h}_{2} \bar{h}_{3} \delta_{2+3}^{k} \mathrm{~d} \boldsymbol{k}_{23} .
\end{aligned}
$$

The left-hand side of (B 2) is proportional to the Fourier transform of the linearized $\mathrm{PV}, \nabla^{2} \bar{h}-\lambda^{-2} \bar{h}$. After applying an inverse Fourier transform, and using the identity $J(h, h)=0$, the equation becomes

$$
\frac{\partial}{\partial \tau}\left(\nabla^{2} \bar{h}-\lambda^{-2} \bar{h}\right)+\lambda J\left(\bar{h}, \nabla^{2} \bar{h}-\lambda^{-2} \bar{h}\right)=0,
$$

which is (3.3), the quasi-geostrophic equation in physical coordinates. 


\section{REFERENCES}

Alford, M. H., Mackinnon, J. A., Zhao, Z., Pinkel, R., Klymak, J. \& Peacock, T. 2007 Internal waves across the Pacific. Geophys. Res. Lett. 34, L24601.

BARTello, P. 1995 Geostrophic adjustment and inverse cascades in rotating stratified turbulence. J. Atmos. Sci. 52, 4410-4428.

Benney, D. J. \& SAffman, P. G. 1966 Nonlinear interactions of random waves in a dispersive medium. Proc. R. Soc. Lond. A 289, 301-320.

Caillol, P. \& Zeitlin, V. 2000 Kinetic equations and stationary energy spectra of weakly nonlinear internal gravity waves. Dyn. Atmos. Oceans 32, 81-112.

Charney, J. G. 1948 On the scale of atmospheric motions. Geophys. Publ. Oslo 17, 3-17.

Dewar, W. K. \& Killworth, P. D. 1995 Do fast gravity waves interact with geostrophic motions? Deep Sea Res. I 42, 1063-1081.

Doodson, A. T. 1922 The harmonic development of the tide-generating potential. Proc. R. Soc. Lond. A 100, 305-329.

DufFy, D. G. 1974 Resonant interactions of inertial-gravity and Rossby waves. J. Atmos. Sci. 31, 1218-1231.

Ford, R., McIntyre, M. E. \& Norton, W. A. 2000 Balance and the slow quasi-manifold: some explicit results. J. Atmos. Sci. 57, 1236-1254.

Garrett, C. J. R. \& Munk, W. H. 1975 Space time scales of internal waves. J. Geophys. Res. 80, 291-299.

Klein, P. \& Llewellyn Smith, S. 2001 Horizontal dispersion of near-inertial oscillations in a turbulent mesoscale eddy field. J. Mar. Res. 59, 697-723.

Klein, P., Llewellyn Smith, S. \& Lapeyre, G. 2004 Organization of near-inertial energy by an eddy field. Q. J. R. Meteorol. Soc. 130, 1153-1166.

KunZE, E. 1985 Near-inertial wave propagation in geostrophic shear. J. Phys. Oceanogr. 15, 544-565.

LeITH, C. E. 1980 Nonlinear normal mode initialization and quasi-geostrophic theory. J. Atmos. Sci. 37, 958-968.

Lelong, M.-P. \& Riley, J. J. 1991 Internal wave-vortical mode interactions in strongly stratified flows. J. Fluid Mech. 232, 1-19.

LoRenZ, E. N. 1980 Attractor sets and quasi-geostrophic equilibrium. J. Atmos. Sci. 37, 1685-1691.

Lvov, Y. V., Polzin, K. L. \& TabaK, E. G. 2004 Energy spectra of the ocean's internal wave field: theory and observation. Phys. Rev. Lett. 92, 128501.

MacKinnon, J. A. \& Winters, K. B. 2005 Subtropical catastrophe: significant loss of low-mode tidal energy at $28.9^{\circ}$. Geophys. Res. Lett. 32, L15605.

McComas, C. H. \& Bretherton, F. P. 1977 Resonant interaction of oceanic internal waves. J. Geophys. Res. 82, 1397-1412.

McComas, C. H. \& Müller, P. 1981 The dynamic balance of internal waves. J. Phys. Oceanogr. 11, 970-986.

McIntyre, M. E. \& Norton, W. A. 2000 Potential vorticity inversion on a hemisphere. J. Atmos. Sci. 57, 1214-1235.

Müller, P., Holloway, G., Henyey, F. \& Pomphrey, N. 1986 Nonlinear interactions among internal gravity waves. Rev. Geophys. 24, 493-536.

Munk, W. \& Wunsch, C. 1998 Abyssal recipes II: energetics of tidal and wind mixing. Deep Sea Res. I 45, 1977-2010.

Oвuкhov, A. M. 1949 On the problem of geostrophic motions (in Russian). Izv. Geogr. Geophys. 13, 281-306.

Olbers, D. J. \& Pomphrey, N. 1981 Disqualifying two candidates for the energy balance of oceanic internal waves. J. Phys. Oceanogr. 11, 1423-1425.

OrsZAG, S. 1971 On the elimination of aliasing in finite-difference schemes by filtering highwavenumber components. J. Atmos. Sci. 28, 1074.

Pedlosky, J. 1987 Geophysical Fluid Dynamics. Springer.

Phillips, O. M. 1960 On the dynamics of unsteady gravity waves of finite amplitude. Part 1. J. Fluid Mech. 9, 193-217.

Polzin, K. L., Toole, J. M., Ledwell, J. R. \& Schmitt, R. W. 1997 Spatial variability of turbulent mixing in the abyssal ocean. Science 276, 93-96. 
Rainville, L. \& Pinkel, R. 2006 Propagation of low-mode internal waves through the ocean. J. Phys. Oceanogr. 36, 1220-1236.

Remmel, M. \& Smith, L. 2009 New intermediate models for rotating shallow water and an investigation of the preference for anticyclones. J. Fluid Mech. 625, 321-359.

Reznik, G. M., Zeitlin, V. \& Ben Jelloul, M. 2001 Nonlinear theory of geostrophic adjustment. Part 1. Rotating shallow-water model. J. Fluid Mech. 445, 93-120.

Simmons, H. L., Hallberg, R. W. \& Arbic, B. K. 2004 Internal wave generation in a global baroclinic tide model. Deep Sea Res. II 51, 3043-3068.

St. Laurent, L. \& Garrett, C. 2002 The role of internal tides in mixing the deep ocean. J. Phys. Oceanogr. 32, 2882-2899.

St. Laurent, L. C. \& Nash, J. D. 2004 An examination of the radiative and dissipative properties of deep ocean internal tides. Deep Sea Res. II 51, 3029-3042.

Waite, M. L. \& Bartello, P. 2006 Stratified turbulence generated by internal gravity waves. J. Fluid Mech. 546, 313-339.

WARN, T. 1986 Statistical mechanical equilibria of the shallow water equations. Tellus 38A, 1-11.

Warn, T., Bokhove, O., Shepard, T. G. \& Vallis, G. K. 1995 Rossby number expansions, slaving principles and balance dynamics. Q. J. R. Meteorol. Soc. 121, 723-739.

Young, W. R. \& Ben Jelloul, M. 1997 Propagation of near-inertial oscillations through a geostrophic flow. J. Mar. Res. 55, 735-766. 